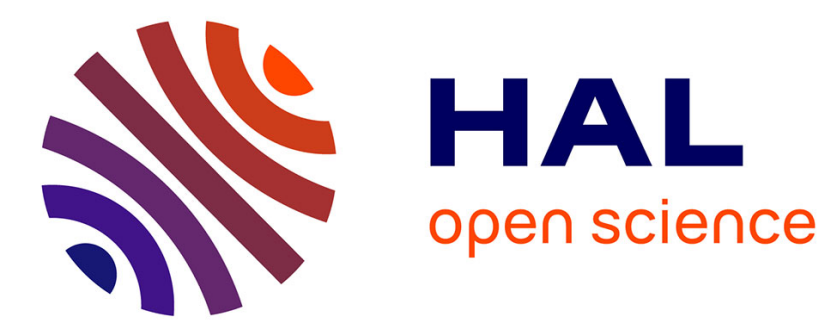

\title{
Volumetric Properties of Binary Mixtures of 1,2-Dichloroethane with Ethers from 278.15-333.15 K and at Atmospheric Pressure
}

Alain Valtz, Esther Neyrolles, Farid Brahim Belaribi, Christophe Coquelet

\section{To cite this version:}

Alain Valtz, Esther Neyrolles, Farid Brahim Belaribi, Christophe Coquelet. Volumetric Properties of Binary Mixtures of 1,2-Dichloroethane with Ethers from 278.15-333.15 K and at Atmospheric Pressure. Journal of Chemical and Engineering Data, 2022, 67 (3), pp.554-567. 10.1021/acs.jced.1c00810 . hal-03574360

\author{
HAL Id: hal-03574360 \\ https://hal.science/hal-03574360
}

Submitted on 15 Feb 2022

HAL is a multi-disciplinary open access archive for the deposit and dissemination of scientific research documents, whether they are published or not. The documents may come from teaching and research institutions in France or abroad, or from public or private research centers.
L'archive ouverte pluridisciplinaire HAL, est destinée au dépôt et à la diffusion de documents scientifiques de niveau recherche, publiés ou non, émanant des établissements d'enseignement et de recherche français ou étrangers, des laboratoires publics ou privés. 


\title{
Volumetric properties of binary mixtures of 1,2-dichloroethane with ethers from $278.15-333.15 \mathrm{~K}$ and at atmospheric pressure
}

\author{
Alain Valtz ${ }^{a}$, Esther Neyrolles ${ }^{a}$, Farid Brahim Belaribi ${ }^{b}$, Christophe Coquelet $^{a, c}$ \\ ${ }^{a}$ Mines ParisTech, PSL University, CTP-Centre of Thermodynamics of Processes, 35 Rue Saint \\ Honoré, 77305 Fontainebleau, France \\ ${ }^{b}$ Thermodynamic and Molecular Modelisation Laboratory, Faculty of Chemistry, University of \\ Sciences and Technology Houari Boumediene (USTHB), B.P. 32 El-Alia, Bab-Ezzouar, Algiers, \\ Algeria \\ ${ }^{c}$ Université de Toulouse, IMT Mines Albi, UMR CNRS 5302, Centre Rapsodee, Campus Jarlard, \\ 81013 Albi CT Cedex 9, France
}

email: christophe.coquelet@mines-albi.fr

\begin{abstract}
Using a vibrating tube density meter, the densities and speed of sound of 1,2-dichloroethane, methyl tert-butyl ether, diisopropyl ether, and diethyl ether were measured between $\mathrm{T}=(278.15$ to 333.15$) \mathrm{K}$ and at atmospheric pressure. The densities of the three binary mixtures: 1,2-dichloroethane + ethers, were also experimentally determined. The Redlich-Kister data treatment and partial molar volume were applied to the excess molar volume of the mixtures. To conclude, the Prigogine-Flory-Patterson model was used to predict the excess molar volume of the three binary systems and to determine the most significant contribution to the excess molar volume.
\end{abstract}

Keywords: Excess molar volume, apparent molar volume, partial molar volume, vibrating densitometer

\section{Introduction}

Thermodynamic properties of a pure compound or multi-compound mixtures are highly required in the context of the chemical and process industry. They are necessary to design, model, and optimize industrial processes, current or new ones. These properties can be experimentally measured or estimated with thermodynamic models or correlations. The 1,2-dichloroethane (DCE), the methyl tertbutyl ether also known as 2-methoxy-2-methylpropane (MTBE), the diisopropyl ether also known as 2-propan-2-yloxypropane (DIPE), and the diethyl ether also known as ethoxyethane (DEE) belongs to two important groups of molecules in chemistry: chloroalkanes and ethers. Both of them are widely used in the chemical industry as a solvent for extraction or chemical reaction, refrigerants, etc., but also in other industries like in the pharmaceutical industry ${ }^{1,2}$. The volumetric properties like density and speed of sound for pure compound and mixing density and excess molar volume for the mixtures are really important in several units operation of the chemical engineering industry such as distillation columns, liquid-liquid extractors, etc. The excess molar volume gives information on the interaction between the molecules in the mixture. The influence of $-\mathrm{O}-$ and $-\mathrm{Cl}$ group interaction between the DCE and the ethers is of interest here. These molecular interactions are essential for the development of appropriate thermodynamic models to design industrial processes. In line with experimental and theoretical previous works ${ }^{3-6}$ on DCE with ether, this study presents the experimental densities of the three mixtures DCE + MTBE, DCE + DIPE, and DCE + DEE between T $=(278.15$ to 333.15) K. In this work, we start by a presentation of the theory considered to correlate the experimental data. Then the experimental apparatus is described. To continue, the results are presented and the excess molar volume estimated is compared with the Redlich-Kister correlation ${ }^{7}$. And lastly, the Prigogine-FloryPatterson (PFP) theory ${ }^{8-11}$ is used to determine the main contribution of the interactions between the molecules. 


\section{Theory section}

The excess molar volume $v^{E}$ of a solution is expressed following Eq. 1:

$$
v^{E}=v-x_{1} v_{1}^{0}-x_{2} v_{2}^{0}
$$

where $x_{1}, x_{2}, v_{1}^{0}$ and $v_{2}^{0}$ represent respectively the mole fractions and molar volumes of DCE (1) and ether (2). Eq. 1 can be rewritten as:

$$
v^{E}=\left[\frac{x_{1} M_{1}+x_{2} M_{2}}{\rho}\right]-\frac{x_{1} M_{1}}{\rho_{1}}-\frac{x_{2} M_{2}}{\rho_{2}}
$$

where $M_{1}, M_{2}, \rho_{1}$ and $\rho_{2}$ represent respectively the molar masses and densities of DCE (1) and ether (2) and $\rho$ represent the density of the mixture.

\subsection{Redlich-Kister correlation}

The Redlich-Kister (RK) correlation ${ }^{7}$ is chosen to correlate excess molar volume of binary systems:

$v^{E}=x_{1} x_{2} \sum_{i} A_{n}\left(x_{1}-x_{2}\right)^{i}$

where $A_{n}$ is the RK coefficient.

The Redlich-Kister coefficients $A_{n}$ are adjusted at each temperature studied. The standard deviation $\sigma$ corresponding to each fit is estimated following Eq 4:

$\sigma=\sqrt{\sum \frac{\left(v^{E}-v_{\text {cal }}^{E}\right)^{2}}{N_{\text {exp }}-P}}$

where $P$ is the number of RK coefficients and $N_{\text {exp }}$ is the number of experimental data. The maximum number of RK coefficients is chosen to have $\sigma$ lower than the excess molar volume uncertainty.

\subsection{Partial molar volume}

The partial molar volume $v_{i}\left(\mathrm{~cm}^{3} \cdot \mathrm{mol}^{-1}\right)$ of each component is calculated following Eq 5:

$v_{i}=\left(\frac{d V}{d n_{i}}\right)_{T, P, n_{j}}$

After differentiation of Eq 1 concerning $n_{i}$ and combining the result to Eq 5. It leads to Eqs 6 and 7 for the partial molar volumes of the different species.

$$
\begin{aligned}
& v_{1}=v^{E}+v_{1}^{0}-x_{2}\left(\frac{d V^{E}}{d x_{2}}\right)_{T, P} \\
& v_{2}=v^{E}+v_{2}^{0}-x_{1}\left(\frac{d V^{E}}{d x_{1}}\right)_{T, P}
\end{aligned}
$$

The expressions of partial molar volumes, for $x_{i}$, are obtained, by using the Redlich-Kister equation, following Eq 8 and 9:

$v_{1}=v_{1}^{0}+x_{2}^{2} \sum A_{n}\left(1-2 x_{2}\right)^{n}+2 x_{2}^{2}\left(1-x_{2}\right) \sum n A_{n}\left(1-2 x_{2}\right)^{n-1}$

$v_{2}=v_{2}^{0}+\left(1-x_{2}\right) \sum A_{n}\left(1-2 x_{2}\right)^{n}-2 x_{2}^{2}\left(1-x_{2}\right) \sum n A_{n}\left(1-2 x_{2}\right)^{n-1}$

At infinite dilution, the partial molar volume is identical to the apparent molar volume $v_{\Phi l}$. The apparent molar volume of DCE in ether $v_{\Phi l}$ is given by Eq. 10: 
$v_{\Phi_{1}}=\frac{v-x_{2} v_{2}^{0}}{x_{1}}$

The combination of Eq. 10 and Eq. 1 leads to Eq. 11 for apparent molar volume $v_{\Phi l}$ of DCE in ether.

$v_{\Phi_{1}}=v_{1}^{0}+\frac{v^{E}}{x_{1}}$

\subsection{Prigogine-Flory-Patterson theory}

The Prigogine-Flory-Patterson (PFP) theory ${ }^{8-11}$ is widely applied to predict excess thermodynamics properties such as the excess molar volume, $v^{E}$. Originally, the Flory model was used to explain the thermodynamic behavior of non-polar mixtures but the model can be used with polar mixture considering Flory parameter adjusted on experimental data following Hansen's theory ${ }^{12}$. In this study, excess properties of the mixtures are linked to the measurable macroscopic properties of the pure constituents.

According to the PFP model, the excess molar volume is the sum of three contributions listed below:

- The interactional contribution $v_{\text {int }}$ is proportional to the empirical Flory parameter $\chi_{12}$ which can be adjusted from experimental excess molar volumes, following Eq 12:

$v_{\text {int }}=\frac{\left(\tilde{v}^{1 / 3}-1\right) \tilde{v}^{2 / 3}}{(4 / 3) \tilde{v}^{-1 / 3}-1} \psi_{1} \theta_{2} \frac{\chi_{12}}{P_{1}^{*}}$

- The free volume contribution $v_{f v}$ is due to the difference between the thermal expansions of the two components, following Eq 13:

$v_{f v}=\frac{\left(\tilde{v}_{1}-\tilde{v}_{2}\right)^{2}\left[(14 / 9) \tilde{v}^{1 / 3}-1\right]}{(4 / 3) \tilde{v}^{-1 / 3}-1} \psi_{1} \psi_{2}$

- The $P^{*}$ contribution (internal pressure contribution) $v_{P^{*}}$ is dependent on both the differences between the reduced volumes and internal pressures of the two molecules, following Eq 14:

$v_{P^{*}}=\frac{\left(\tilde{v}_{1}-v\right)^{2}\left(P_{1}^{*}-P_{2}^{*}\right)}{P_{1}^{*} * \psi_{2}+P_{2}^{*} * \psi_{1}} * \psi_{1} \psi_{2}$

$\tilde{v}$ is the reduced volume $\left(\widetilde{v}=\psi_{1} \tilde{v}_{1}+\psi_{2} \tilde{v}_{2}\right), \psi_{i}$ is the molecular contact energy $\left(\psi_{1}=1-\psi_{2}=\right.$ $\left.\frac{\varphi_{1} P_{1}^{*}}{\varphi_{1} P_{1}^{*}+\varphi_{2} P_{2}^{*}}\right)$. The hardcore volume fraction $\varphi_{i}$ is given by $\varphi_{1}=1-\varphi_{2}=\frac{x_{1} v_{1}^{*}}{x_{1} v_{1}^{*}+x_{2} v_{2}^{*}}$.

$\theta_{2}$ is the molecular surface fraction $\left(\theta_{2}=\frac{\varphi_{2}}{\varphi_{1}\left(S_{1} / S_{2}\right)+\varphi_{2}}\right)$ with the ratio $S_{1} / S_{2}$ approximates by $\left(V_{2}{ }^{*} / V_{l}^{*}\right)^{1 / 3}$ according to Abe and Flory ${ }^{10}$.

$\tilde{v}_{i}$ is the reduced volume defined in $\mathrm{Eq} 15$ :

$\tilde{v}_{i}=\left[\frac{1+\left(\frac{4}{3}\right) \alpha_{i} T}{1+\alpha_{i} T}\right]^{3}$

The thermal coefficient expansion $\alpha_{i}$ is given by $\alpha_{i}=\left(1 / \mathrm{v}_{i}^{0}\right)\left(\partial \mathrm{v}_{i}^{0} / \partial \mathrm{T}\right)_{P} \cdot v_{i}{ }^{*}$ and $P_{i}{ }^{*}$ are respectively the characteristic volume $\left(v_{i}^{*}=\frac{v_{i}}{\tilde{v}_{i}}\right)$ and pressure $\left(P_{i}^{*}=\frac{T \tilde{v}_{i}{ }^{2} \alpha_{i}}{\kappa_{T i}}\right)$.

The isothermal compressibility $\kappa_{T i}$ is given by $\kappa_{T i}=-(1 / \mathrm{v})(\partial \mathrm{v} / \partial \mathrm{T})_{T}$

The isothermal compressibility $\kappa_{T i}$ is also related via Maxwell's relation (Eq. 16) to the isentropic compressibility $\kappa_{S}$. 
$\kappa_{T i}=\kappa_{s i}+\alpha_{i}^{2} V_{i} \frac{T}{C_{p i}}$

where $\mathrm{V}_{i}$ is the molar volume of the compound $i$ and $C_{p i}$ is the liquid heat capacity. The isentropic compressibility $\kappa_{s i}$ can be estimated using the density $\rho_{i}$ and the speed of sound $u_{i}$ of a compound $\left(\mathrm{u}_{i}=\sqrt{\frac{1}{\kappa_{s i} \rho_{i}}}\right)$.

Consequently, in the terms of the three contributions to the PFP theory, the excess molar volume is given by Eq. 17:

$$
\begin{aligned}
\frac{{ }^{E}}{x_{1} v_{1}^{*}+\chi_{2} v_{2}^{*}}=v_{i n t}+v_{f v}+v_{P^{*}}= & \frac{\left(\frac{1}{\tilde{v}^{3}}-1\right) \tilde{v}^{\frac{2}{3}}}{\left(\frac{4}{3}\right) \tilde{v}^{-\frac{1}{3}}-1} \psi_{1} \theta_{2} \frac{\chi_{12}}{P_{1}^{*}}+\frac{\left(\tilde{v}_{1}-\tilde{v}_{2}\right)^{2}\left[\left(\frac{14}{9}\right) \tilde{v}^{\frac{1}{3}}-1\right]}{\left.\left(\frac{4}{3}\right)\right)^{-\frac{1}{3}}-1} \psi_{1} \psi_{2} \\
& +\frac{\left(\tilde{v}_{1}-\tilde{v}_{2}\right)^{2}\left(P_{1}^{*}-P_{2}^{*}\right)}{P_{1}^{*} \psi_{2}+P_{2}^{*} \psi_{1}} \psi_{1} \psi_{2}
\end{aligned}
$$

\section{Experimental section}

\subsection{Chemicals}

The list of chemical species used is presented in Table 1, with the suppliers and the purities. Chemicals were used without any further purification.

\begin{tabular}{|c|c|c|c|c|c|}
\hline Chemical name & Abbreviation & $\begin{array}{c}\text { CAS } \\
\text { number }\end{array}$ & Source & $\begin{array}{c}\text { Mole Fraction } \\
\text { Purity reported by } \\
\text { the supplier }\end{array}$ & $\begin{array}{l}\text { Analysis } \\
\text { Method }\end{array}$ \\
\hline 1,2-dichloroethane & DCE & $107-06-2$ & $\begin{array}{c}\text { Acros } \\
\text { organics }\end{array}$ & 0.998 & $\mathrm{GC}^{\mathrm{a}}$ \\
\hline $\begin{array}{l}\text { 2-methoxy-2- } \\
\text { methylpropane }\end{array}$ & MTBE & $1634-04-4$ & $\begin{array}{c}\text { Acros } \\
\text { organics }\end{array}$ & 0.999 & $\mathrm{GC}^{\mathrm{a}}$ \\
\hline 2-propan-2-yloxypropane & DIPE & $108-20-3$ & Merck & 0.985 & $\mathrm{GC}^{\mathrm{a}}$ \\
\hline ethoxyethane & DEE & $60-29-7$ & $\begin{array}{c}\text { Acros } \\
\text { organics }\end{array}$ & 0.990 & $\mathrm{GC}^{\mathrm{a}}$ \\
\hline
\end{tabular}

Table 1: Chemical sample

${ }^{\mathrm{a}} \mathrm{GC}$ : Gas chromatograph

\subsection{Apparatus}

Density measurements have been made with the DSA5000M Anton Paar digital vibrating tube density meter. The experimental equipment has already been described in the previous studies ${ }^{3,13,14}$, only a few essentials are given here. The tube mass of the density meter and so the fluid density are dependent of the oscillating period or frequency. The period of vibration $\tau$ is related to density $\rho_{\mathrm{i}}$ following Eq. 18, where $a$ and $b$ are constants to be adjusted.

$\rho_{i}=a+b \tau^{2}$

The density meter was checked at $T=293.15 \mathrm{~K}$ with dry air and de-gassed bi-distilled ultrapure water. The temperature is measured with a platinum resistance thermometer $(0.01 \mathrm{~K}$ accuracy). When the density meter is at thermal equilibrium, the sample densities are measured. Mixtures were prepared by weighing, with uncertainty in mole fraction estimated to be less than 0.002 . All weighing performed in the experimental work was carried out using a Mettler balance, with a precision of \pm 
$1.10^{-4} \mathrm{~g}$. As recommended by Chirico and al. ${ }^{15}$, the purities of each compound $x_{i s}$, presented in Table 1 , are taken into account for the estimation of the expanded uncertainties. The maximum uncertainty on measured density is estimated to be: $\mathrm{u}\left(\rho_{i}\right)=1.5 \mathrm{~kg} . \mathrm{m}^{-3}$. For the excess molar volumes, the maximum $\mathrm{u}\left(v^{E}\right)$ is estimated to be $\pm 0.011 \mathrm{~cm}^{3} \cdot \mathrm{mol}^{-1}$.

To estimate the uncertainty of the mole fraction $\mathrm{u}\left(x_{i}\right)$, the density $\mathrm{u}\left(\rho_{i}\right)$ of the compound $\mathrm{i}$, the density of the mixture $\mathrm{u}(\rho)$ and the excess molar volume $\mathrm{u}\left(v^{E}\right)$, the following Eqs 19 to 22 were used:

$$
\begin{aligned}
& u\left(\rho_{i}\right)=\xi\left(1-x_{s i}\right) \\
& u\left(x_{i}\right)=x_{1} x_{2}\left(1-x_{s 1}\right)\left(1-x_{s 2}\right) \sqrt{\frac{1}{\left(m_{1}\right)^{2}}+\frac{1}{\left(m_{2}\right)^{2}}} \\
& u(\rho)=\sqrt{u\left(\rho_{1}\right)^{2}+u\left(\rho_{2}\right)^{2}+u\left(x_{i}\right)^{2}} \\
& u\left(v^{E}\right)=\sqrt{\left[\left(\frac{x_{1} M_{1}+x_{2} M_{2}}{\rho^{2}}\right)^{2}+\left(\frac{x_{1} M_{1}}{\rho_{1}{ }^{2}}\right)^{2}+\left(\frac{x_{2} M_{2}}{\rho_{2}{ }^{2}}\right)^{2}\right] u(\rho)^{2}+\left(\frac{M_{1}+M_{2}}{\rho}-\frac{M_{1}}{\rho_{1}}-\frac{M_{2}}{\rho_{2}}\right)^{2} u\left(x_{i}\right)^{2}}
\end{aligned}
$$

where $\xi$ is the presumed difference in density between the compound and the impurity. We have chose to use $\xi=0.1$ as recommended by Chirico and al. ${ }^{15}$.

This density meter is also used to measure the speed of sound $u_{i}$ with an uncertainty of $0.5 \mathrm{~m} . \mathrm{s}^{-1}$.

The density meter is not equipped with a pressure transducer. In consequence, the value of atmospheric pressure is given by a GE Druck DPI 142, Precision Barometric Indicator with standard uncertainty $\mathrm{u}(p)=0.035 \mathrm{kPa}$. We have considered as uncertainty on atmospheric pressure the variation of atmospheric pressure during the experiment which was lower than $0.1 \mathrm{kPa}$.

\section{Results}

The densities and the speed of sound of the four pure compounds measured by the DSA 5000M Anton Paar density meter, between $\mathrm{T}=(278.15$ to 333.15$) \mathrm{K}$ and at atmospheric pressure, are presented in Table 2. The experimental data concerning the densities and the speed of sound of DCE where already published by Valtz et al. ${ }^{3}$. In appendix, we have compared our experimental data with literature data.

For the DCE + MTBE, DCE + DIPE, DCE + DEE binary mixtures, the values of mixture densities and excess molar volumes are shown respectively in Table 3 to 5. The Redlich-Kister parameters and deviation for three binary systems are presented in Erreur! Source du renvoi introuvable. to S3 (in supporting Information). The number of Redlich-Kister parameters is selected according to the method of Desnoyers and Perron ${ }^{16}$. At all temperatures, $v^{E} / x_{1} x_{2}$ as a function of $x_{1}$ and $v^{E}$ as a function of $\mathrm{x}_{1}$ are represented, respectively, in Figure 1 and Figure 2 for the DCE + MTBE system, in Figure 3 and Figure 4 for the DCE + DIPE system, and in Figure 5 and Figure 6 for the DCE + DEE system. 
Table 2: Experimental densities $\rho_{i}$ and speed of sound $u_{i}$ of the four compounds at different temperatures $T$ and atmospheric pressure $(p=101.33 \mathrm{kPa})$.

\begin{tabular}{|c|c|c|c|c|c|c|c|c|}
\hline \multirow{2}{*}{$T / \mathbf{K}$} & \multicolumn{2}{|c|}{$\mathrm{DCE}^{\mathrm{a}}$} & \multicolumn{2}{|c|}{ DIPE } & \multicolumn{2}{|c|}{ MTBE } & \multicolumn{2}{|c|}{ DEE } \\
\hline & $\rho / \mathrm{kg} \mathrm{m}^{-3}$ & $u / \mathrm{m} . \mathrm{s}^{-1}$ & $\rho / \mathrm{kg} \mathrm{m}^{-3}$ & $u / \mathrm{m} . \mathrm{s}^{-1}$ & $\rho / \mathrm{kg} \mathrm{m}^{-3}$ & $u / \mathrm{m}_{\mathrm{s}} \mathrm{s}^{-1}$ & $\rho / \mathrm{kg}^{-\mathrm{m}^{-3}}$ & $u / \mathrm{m} . \mathrm{s}^{-1}$ \\
\hline 278.14 & 1274.6 & 1271.2 & 739.1 & 1088.1 & 756.1 & 1128.9 & 730.8 & 1075.4 \\
\hline 279.15 & 1273.2 & 1267.5 & 738.0 & 1083.8 & 755.1 & 1124.4 & 729.7 & 1070.9 \\
\hline 280.15 & 1271.7 & 1263.3 & 737.0 & 1078.9 & 754.1 & 1119.3 & 728.6 & 1065.8 \\
\hline 281.15 & 1270.3 & 1259.7 & 736.0 & 1074.7 & 753.0 & 1115.0 & 727.4 & 1061.4 \\
\hline 282.15 & 1268.8 & 1255.4 & 735.0 & 1069.7 & 752.0 & 1109.8 & 726.3 & 1056.2 \\
\hline 283.15 & 1267.4 & 1251.9 & 733.9 & 1065.5 & 751.0 & 1105.6 & 725.1 & 1051.8 \\
\hline 284.15 & 1266.0 & 1247.6 & 732.9 & 1060.6 & 749.9 & 1100.4 & 724.0 & 1046.5 \\
\hline 285.14 & 1264.5 & 1244.0 & 731.9 & 1056.4 & 748.9 & 1096.2 & 722.9 & 1042.2 \\
\hline 286.15 & 1263.1 & 1239.7 & 730.9 & 1051.4 & 747.9 & 1090.9 & 721.7 & 1037.0 \\
\hline 287.15 & 1261.6 & 1236.2 & 729.8 & 1047.3 & 746.9 & 1086.7 & 720.6 & 1032.6 \\
\hline 288.15 & 1260.2 & 1231.8 & 728.8 & 1042.3 & 745.8 & 1081.5 & 719.4 & 1027.3 \\
\hline 289.15 & 1258.7 & 1228.3 & 727.8 & 1038.2 & 744.8 & 1077.3 & 718.3 & 1023.0 \\
\hline 290.15 & 1257.3 & 1224.0 & 726.7 & 1033.2 & 743.7 & 1072.0 & 717.1 & 1017.6 \\
\hline 291.15 & 1255.8 & 1220.6 & 725.7 & 1029.2 & 742.7 & 1067.9 & 715.9 & 1013.5 \\
\hline 292.15 & 1254.4 & 1216.2 & 724.7 & 1024.2 & 741.7 & 1062.8 & 714.8 & 1008.2 \\
\hline 293.15 & 1252.9 & 1212.8 & 723.6 & 1020.3 & 740.6 & 1058.7 & 713.6 & 1004.0 \\
\hline 294.15 & 1251.4 & 1208.5 & 722.6 & 1015.3 & 739.6 & 1053.6 & 712.4 & 998.7 \\
\hline 295.15 & 1250.0 & 1205.1 & 721.5 & 1011.3 & 738.5 & 1049.5 & 711.2 & 994.5 \\
\hline 296.15 & 1248.5 & 1200.7 & 720.5 & 1006.4 & 737.5 & 1044.3 & 710.1 & 989.2 \\
\hline 297.14 & 1247.1 & 1197.3 & 719.5 & 1002.3 & 736.4 & 1040.1 & 708.9 & 984.9 \\
\hline 298.15 & 1245.6 & 1193.0 & 718.4 & 997.7 & 735.4 & 1035.4 & 707.7 & 980.0 \\
\hline 299.15 & 1244.2 & 1189.6 & 717.4 & 993.4 & 734.3 & 1030.8 & 706.5 & 975.4 \\
\hline 300.15 & 1242.7 & 1185.6 & 716.3 & 988.9 & 733.3 & 1026.2 & 705.4 & 970.5 \\
\hline 301.15 & 1241.2 & 1181.8 & 715.3 & 984.4 & 732.2 & 1021.5 & 704.2 & 965.7 \\
\hline 302.15 & 1239.8 & 1177.9 & 714.2 & 980.0 & 731.1 & 1016.9 & 703.0 & 961.0 \\
\hline 303.14 & 1238.3 & 1174.0 & 713.1 & 975.6 & 730.1 & 1012.3 & 701.8 & 956.2 \\
\hline 304.14 & 1236.8 & 1170.2 & 712.1 & 971.1 & 729.0 & 1007.7 & 700.6 & 951.5 \\
\hline 305.14 & 1235.4 & 1166.3 & 711.0 & 966.7 & 727.9 & 1003.0 & 699.4 & 946.8 \\
\hline 306.15 & 1233.9 & 1162.4 & 710.0 & 962.2 & 726.9 & 998.4 & 698.2 & 942.0 \\
\hline 307.15 & 1232.4 & 1158.6 & 708.9 & 957.8 & 725.8 & 993.8 & 697.0 & 937.3 \\
\hline 308.15 & 1231.0 & 1155.1 & 707.8 & 953.4 & 724.7 & 989.2 & & \\
\hline 309.15 & 1229.5 & 1150.9 & 706.8 & 949.0 & 723.7 & 984.6 & & \\
\hline 310.15 & 1228.0 & 1147.1 & 705.7 & 944.6 & 722.6 & 980.0 & & \\
\hline 311.15 & 1226.5 & 1143.2 & 704.6 & 940.3 & 721.5 & 975.4 & & \\
\hline 312.15 & 1225.1 & 1139.4 & 703.6 & 935.9 & 720.4 & 970.8 & & \\
\hline 313.15 & 1223.6 & 1135.3 & 702.5 & 931.1 & 719.3 & 966.2 & & \\
\hline 314.15 & 1222.1 & 1131.7 & 701.4 & 927.2 & 718.2 & 961.6 & & \\
\hline 315.15 & 1220.6 & 1127.9 & 700.3 & 922.8 & 717.1 & 957.0 & & \\
\hline 316.15 & 1219.1 & 1124.1 & 699.3 & 918.4 & 716.1 & 952.5 & & \\
\hline 317.15 & 1217.6 & 1120.3 & 698.2 & 914.0 & 715.0 & 947.9 & & \\
\hline 318.15 & 1216.2 & 1116.2 & 697.1 & 909.3 & 713.9 & 943.1 & & \\
\hline 319.14 & 1214.7 & 1112.6 & 696.0 & 905.2 & 712.8 & 938.7 & & \\
\hline 320.15 & 1213.2 & 1108.8 & 694.9 & 900.8 & 711.7 & 934.1 & & \\
\hline 321.15 & 1211.7 & 1105.0 & 693.8 & 896.5 & 710.6 & 929.6 & & \\
\hline 322.15 & 1210.2 & 1101.2 & 692.7 & 892.2 & 709.4 & 925.0 & & \\
\hline 323.14 & 1208.7 & 1097.2 & 691.6 & 887.9 & 708.3 & 920.5 & & \\
\hline
\end{tabular}




\begin{tabular}{lllllll}
$\mathbf{3 2 4 . 1 5}$ & 1207.2 & 1093.6 & 690.5 & 883.6 & 707.2 & 915.9 \\
$\mathbf{3 2 5 . 1 5}$ & 1205.7 & 1089.8 & 689.4 & 879.2 & 706.1 & 911.4 \\
$\mathbf{3 2 6 . 1 4}$ & 1204.2 & 1086.0 & 688.3 & 874.9 & 705.0 & 906.9 \\
$\mathbf{3 2 7 . 1 5}$ & 1202.7 & 1082.2 & 687.2 & 870.6 & 703.9 & 902.4 \\
$\mathbf{3 2 8 . 1 4}$ & 1201.2 & 1078.2 & 686.1 & 866.3 & 702.7 & 897.9 \\
$\mathbf{3 2 9 . 1 5}$ & 1199.7 & 1074.6 & 685.0 & 862.0 & 701.6 & 893.4 \\
$\mathbf{3 3 0 . 1 4}$ & 1198.2 & 1070.9 & 683.9 & 857.7 & & \\
$\mathbf{3 3 1 . 1 4}$ & 1196.7 & 1067.1 & 682.8 & 853.4 & & \\
$\mathbf{3 3 2 . 1 5}$ & 1195.2 & 1063.3 & 681.7 & 849.1 & & \\
$\mathbf{3 3 3 . 1 4}$ & 1193.7 & 1059.3 & 680.6 & 844.7 & & \\
$\mathbf{3 3 4 . 1 4}$ & 1192.2 & 1055.7 & 679.4 & 840.4 & & \\
$\mathbf{3 3 5 . 1 5}$ & 1190.6 & 1052.0 & 678.3 & 836.1 & & \\
$\mathbf{3 3 6 . 1 5}$ & 1189.1 & 1048.2 & 677.2 & 831.8 & & \\
$\mathbf{3 3 7 . 1 5}$ & 1187.6 & 1044.4 & 676.1 & 827.5 & & \\
$\mathbf{3 3 8 . 1 5}$ & 1186.1 & 1040.6 & 674.9 & 823.3 & & \\
$\mathbf{3 3 9 . 1 5}$ & 1184.6 & 1036.9 & 673.8 & 819.0 & & \\
$\mathbf{3 4 0 . 1 4}$ & 1183.0 & 1033.2 & 672.7 & 814.8 & & \\
$\mathbf{3 4 1 . 1 5}$ & 1181.5 & 1029.4 & 671.5 & 810.6 & & \\
$\mathbf{3 4 2 . 1 4}$ & 1180.0 & 1025.6 & & & & \\
\hline
\end{tabular}

${ }^{\mathrm{a}}$ : These data were already published by Valtz et al. [3] in 2011

Standard uncertainty $\mathrm{u}(p)=0.1 \mathrm{kPa}, \mathrm{u}(T)=0.01 \mathrm{~K}$

Expanded uncertainties $(\mathrm{k}=2) \mathrm{U}\left(\rho_{i}\right)=1.5 \mathrm{~kg} \cdot \mathrm{m}^{-3}, \mathrm{U}\left(u_{\mathrm{i}}\right)=0.5 \mathrm{~m} \cdot \mathrm{s}^{-1}$ 
Table 3: DCE (1) + MTBE (2) binary mixture densities $\rho$ and excess molar volumes $v^{E}$ as a function of mole fraction in DCE $x_{1}$ at different temperatures $T$ and atmospheric pressure $(p=101.33 \mathrm{kPa})$.

\begin{tabular}{|c|c|c|c|c|c|c|c|c|c|c|}
\hline \multirow{2}{*}{$x_{1}^{T / K}$} & \multicolumn{2}{|c|}{278.15} & \multicolumn{2}{|c|}{283.15} & \multicolumn{2}{|c|}{288.15} & \multicolumn{2}{|c|}{293.15} & \multicolumn{2}{|c|}{298.15} \\
\hline & $\rho / \mathbf{k g} \cdot \mathrm{m}^{-3}$ & $v^{E} / \mathrm{cm}^{3} \cdot \mathrm{mol}^{-1}$ & $\rho / \mathbf{k g} \cdot \mathbf{m}^{-3}$ & $v^{E} / \mathrm{cm}^{3} \cdot \mathrm{mol}^{-1}$ & $\rho / \mathbf{k g} \cdot \mathrm{m}^{-3}$ & $v^{E} / \mathrm{cm}^{3} \cdot \mathrm{mol}^{-1}$ & $\rho / \mathbf{k g} \cdot \mathrm{m}^{-3}$ & $v^{E} / \mathrm{cm}^{3} \cdot \mathrm{mol}^{-1}$ & $\rho / \mathbf{k g}^{-m^{-3}}$ & $v^{E} / \mathrm{cm}^{3} \cdot \mathrm{mol}^{-1}$ \\
\hline 1.000 & 1274.6 & 0.000 & 1267.4 & 0.000 & 1260.2 & 0.000 & 1252.9 & 0.000 & 1245.6 & 0.000 \\
\hline 0.975 & 1256.1 & -0.043 & 1248.9 & -0.045 & 1241.8 & -0.047 & 1234.6 & -0.050 & 1227.4 & -0.051 \\
\hline 0.950 & 1237.8 & -0.078 & 1230.8 & -0.082 & 1223.7 & -0.085 & 1216.6 & -0.089 & 1209.5 & -0.093 \\
\hline 0.900 & 1202.9 & -0.150 & 1196.0 & -0.157 & 1189.0 & -0.164 & 1182.1 & -0.170 & 1175.1 & -0.178 \\
\hline 0.859 & 1175.0 & -0.205 & 1168.2 & -0.213 & 1161.3 & -0.223 & 1154.5 & -0.233 & 1147.6 & -0.243 \\
\hline 0.800 & 1136.7 & -0.272 & 1130.1 & -0.284 & 1123.4 & -0.297 & 1116.7 & -0.309 & 1110.0 & -0.323 \\
\hline 0.749 & 1105.1 & -0.329 & 1098.6 & -0.343 & 1092.1 & -0.358 & 1085.5 & -0.373 & 1078.9 & -0.390 \\
\hline 0.700 & 1076.1 & -0.371 & 1069.7 & -0.388 & 1063.3 & -0.406 & 1056.8 & -0.422 & 1050.4 & -0.441 \\
\hline 0.650 & 1047.2 & -0.413 & 1041.0 & -0.431 & 1034.7 & -0.449 & 1028.3 & -0.468 & 1021.9 & -0.488 \\
\hline 0.600 & 1020.2 & -0.453 & 1014.0 & -0.473 & 1007.8 & -0.492 & 1001.6 & -0.511 & 995.3 & -0.532 \\
\hline 0.527 & 981.9 & -0.502 & 975.9 & -0.522 & 969.8 & -0.543 & 963.7 & -0.563 & 957.6 & -0.586 \\
\hline 0.500 & 968.4 & -0.502 & 962.4 & -0.523 & 956.4 & -0.543 & 950.4 & -0.562 & 944.3 & -0.585 \\
\hline 0.476 & 956.6 & -0.520 & 950.7 & -0.539 & 944.7 & -0.560 & 938.7 & -0.580 & 932.7 & -0.604 \\
\hline 0.450 & 944.0 & -0.512 & 938.1 & -0.533 & 932.2 & -0.553 & 926.3 & -0.573 & 920.3 & -0.594 \\
\hline 0.414 & 926.9 & -0.522 & 921.1 & -0.542 & 915.2 & -0.563 & 909.3 & -0.581 & 903.4 & -0.604 \\
\hline 0.350 & 897.4 & -0.507 & 891.7 & -0.525 & 886.0 & -0.543 & 880.2 & -0.561 & 874.4 & -0.584 \\
\hline 0.323 & 885.4 & -0.509 & 879.8 & -0.527 & 874.1 & -0.545 & 868.4 & -0.561 & 862.7 & -0.584 \\
\hline 0.250 & 853.9 & -0.445 & 848.4 & -0.462 & 842.9 & -0.478 & 837.3 & -0.491 & 831.7 & -0.511 \\
\hline 0.200 & 833.1 & -0.390 & 827.7 & -0.403 & 822.2 & -0.415 & 816.7 & -0.429 & 811.1 & -0.446 \\
\hline 0.150 & 813.0 & -0.319 & 807.6 & -0.331 & 802.2 & -0.340 & 796.8 & -0.350 & 791.3 & -0.366 \\
\hline 0.098 & 792.5 & -0.226 & 787.2 & -0.235 & 781.9 & -0.242 & 776.6 & -0.248 & 771.2 & -0.258 \\
\hline 0.050 & 774.5 & -0.116 & 769.3 & -0.120 & 764.1 & -0.126 & 758.8 & -0.127 & 753.5 & -0.133 \\
\hline 0.025 & 765.3 & -0.063 & 760.1 & -0.068 & 754.9 & -0.071 & 749.7 & -0.072 & 744.4 & -0.077 \\
\hline 0.000 & 756.1 & 0.000 & 751.0 & 0.000 & 745.8 & 0.000 & 740.6 & 0.000 & 735.4 & 0.000 \\
\hline
\end{tabular}


Table 3 (continued)

\begin{tabular}{|c|c|c|c|c|c|c|c|c|c|c|}
\hline \multirow{2}{*}{$x_{x_{1}}$} & \multicolumn{2}{|c|}{303.15} & \multicolumn{2}{|c|}{308.15} & \multicolumn{2}{|c|}{313.15} & \multicolumn{2}{|c|}{318.15} & \multicolumn{2}{|c|}{323.15} \\
\hline & $\rho / \mathbf{k g} \cdot \mathrm{m}^{-3}$ & $v^{E} / \mathrm{cm}^{3} \cdot \mathrm{mol}^{-1}$ & $\rho / \mathrm{kg} \mathrm{m}^{-3}$ & $v^{E} / \mathrm{cm}^{3} \cdot \mathrm{mol}^{-1}$ & $\rho / \mathbf{k g} \cdot \mathrm{m}^{-3}$ & $v^{E} / \mathrm{cm}^{3} \cdot \mathrm{mol}^{-1}$ & $\rho / \mathbf{k g} \cdot \mathrm{m}^{-3}$ & $v^{E} / \mathrm{cm}^{3} \cdot \mathrm{mol}^{-1}$ & $\rho / \mathrm{kg} \cdot \mathrm{m}^{-3}$ & $v^{E} / \mathrm{cm}^{3} \cdot \mathrm{mol}^{-1}$ \\
\hline 1.000 & 1238.3 & 0.000 & 1231.0 & 0.000 & 1223.6 & 0.000 & 1216.2 & 0.000 & 1208.7 & 0.000 \\
\hline 0.975 & 1220.2 & -0.054 & 1212.9 & -0.055 & 1205.6 & -0.057 & 1198.2 & -0.059 & 1190.8 & -0.063 \\
\hline 0.950 & 1202.3 & -0.097 & 1195.1 & -0.101 & 1187.8 & -0.106 & 1180.6 & -0.109 & 1173.3 & -0.116 \\
\hline 0.900 & 1168.0 & -0.187 & 1161.0 & -0.194 & 1153.9 & -0.201 & 1146.7 & -0.211 & 1139.6 & -0.222 \\
\hline 0.859 & 1140.7 & -0.255 & 1133.7 & -0.265 & 1126.7 & -0.277 & 1119.7 & -0.290 & 1112.6 & -0.304 \\
\hline 0.800 & 1103.2 & -0.338 & 1096.4 & -0.351 & 1089.5 & -0.367 & 1082.7 & -0.389 & 1075.7 & -0.404 \\
\hline 0.749 & 1072.2 & -0.407 & 1065.5 & -0.422 & 1058.8 & -0.443 & 1052.1 & -0.463 & 1045.3 & -0.485 \\
\hline 0.700 & 1043.8 & -0.459 & 1037.2 & -0.478 & 1030.6 & -0.501 & 1024.0 & -0.524 & 1017.3 & -0.548 \\
\hline 0.650 & 1015.5 & -0.508 & 1009.1 & -0.531 & 1002.6 & -0.556 & 996.0 & -0.580 & 989.4 & -0.607 \\
\hline 0.600 & 989.0 & -0.554 & 982.6 & -0.579 & 976.2 & -0.604 & 969.8 & -0.630 & 963.3 & -0.658 \\
\hline 0.527 & 951.4 & -0.610 & 945.2 & -0.634 & 939.0 & -0.661 & 932.7 & -0.688 & 926.3 & -0.718 \\
\hline 0.500 & 938.2 & -0.610 & 932.0 & -0.635 & 925.8 & -0.661 & 919.6 & -0.688 & 913.3 & -0.719 \\
\hline 0.476 & 926.7 & -0.629 & 920.5 & -0.652 & 914.4 & -0.680 & 908.1 & -0.704 & 901.9 & -0.737 \\
\hline 0.450 & 914.3 & -0.619 & 908.2 & -0.644 & 902.1 & -0.671 & 895.9 & -0.698 & 889.7 & -0.728 \\
\hline 0.414 & 897.5 & -0.629 & 891.5 & -0.653 & 885.4 & -0.680 & 879.3 & -0.707 & 873.2 & -0.737 \\
\hline 0.350 & 868.6 & -0.607 & 862.7 & -0.630 & 856.7 & -0.655 & 850.7 & -0.680 & 844.7 & -0.708 \\
\hline 0.323 & 856.9 & -0.607 & 851.0 & -0.628 & 845.1 & -0.654 & 839.2 & -0.679 & 833.2 & -0.706 \\
\hline 0.250 & 826.0 & -0.531 & 820.3 & -0.549 & 814.5 & -0.572 & 808.6 & -0.592 & 802.8 & -0.616 \\
\hline 0.200 & 805.5 & -0.463 & 799.9 & -0.478 & 794.2 & -0.497 & 788.4 & -0.516 & 782.6 & -0.537 \\
\hline 0.150 & 785.8 & -0.379 & 780.3 & -0.391 & 774.6 & -0.407 & 768.9 & -0.420 & 763.2 & -0.436 \\
\hline 0.098 & 765.7 & -0.268 & 760.3 & -0.276 & 754.7 & -0.287 & 749.1 & -0.298 & 743.5 & -0.310 \\
\hline 0.050 & 748.1 & -0.140 & 742.7 & -0.144 & 737.2 & -0.151 & 731.7 & -0.155 & 726.1 & -0.163 \\
\hline 0.025 & 739.1 & -0.080 & 733.7 & -0.080 & 728.3 & -0.086 & 722.8 & -0.088 & 717.2 & -0.092 \\
\hline 0.000 & 731.1 & 0.000 & 724.7 & 0.000 & 719.3 & 0.000 & 713.9 & 0.000 & 708.3 & 0.000 \\
\hline
\end{tabular}

Standard uncertainty $\mathrm{u}(p)=0.1 \mathrm{kPa}, \mathrm{u}(T)=0.01 \mathrm{~K}, \mathrm{u}\left(x_{l}\right)=0.002$

Expanded uncertainties $(\mathrm{k}=2) \mathrm{U}(\rho)=1.7 \mathrm{~g} \cdot \mathrm{cm}^{-3}, \mathrm{U}\left(\mathrm{v}^{E}\right)=0.011 \mathrm{~cm}^{3} \cdot \mathrm{mol}^{-1}$ 
Table 4: DCE (1) + DIPE (2) binary mixture densities $\rho$ and excess molar volumes $\mathbf{v}^{\mathrm{E}}$ as a function of mole fraction in DCE $\boldsymbol{x}_{1}$ at different temperatures $T$ and atmospheric pressure $(p=101.33 \mathrm{kPa})$.

\begin{tabular}{|c|c|c|c|c|c|c|c|c|c|c|}
\hline \multirow{2}{*}{$x_{x_{1}}$} & \multicolumn{2}{|c|}{283.15} & \multicolumn{2}{|c|}{288.15} & \multicolumn{2}{|c|}{293.15} & \multicolumn{2}{|c|}{298.15} & \multicolumn{2}{|c|}{303.15} \\
\hline & $\rho / \mathbf{k g} \cdot \mathrm{m}^{-3}$ & $v^{E} / \mathrm{cm}^{3} \cdot \mathrm{mol}^{-1}$ & $\rho / \mathbf{k g} \cdot \mathrm{m}^{-3}$ & $v^{E} / \mathrm{cm}^{3} \cdot \mathrm{mol}^{-1}$ & $\rho / \mathbf{k g} \cdot \mathbf{m}^{-3}$ & $v^{E} / \mathrm{cm}^{3} \cdot \mathrm{mol}^{-1}$ & $\rho / \mathbf{k g} \cdot \mathbf{m}^{-3}$ & $v^{E} / \mathrm{cm}^{3} \cdot \mathrm{mol}^{-1}$ & $\rho / \mathbf{k g} \cdot \mathbf{m}^{-3}$ & $v^{E} / \mathrm{cm}^{3} \cdot \mathrm{mol}^{-1}$ \\
\hline 1.000 & 1267.4 & 0.000 & 1260.2 & 0.000 & 1252.9 & 0.000 & 1245.6 & 0.000 & 1238.3 & 0.000 \\
\hline 0.980 & 1249.7 & -0.082 & 1242.5 & -0.084 & 1235.3 & -0.086 & 1228.1 & -0.088 & 1220.8 & -0.090 \\
\hline 0.960 & 1232.6 & -0.136 & 1225.5 & -0.141 & 1218.4 & -0.144 & 1211.2 & -0.147 & 1204.0 & -0.150 \\
\hline 0.940 & 1215.7 & -0.187 & 1208.6 & -0.192 & 1201.6 & -0.198 & 1194.4 & -0.200 & 1187.3 & -0.206 \\
\hline 0.920 & 1199.2 & -0.235 & 1192.2 & -0.242 & 1185.2 & -0.249 & 1178.2 & -0.255 & 1171.1 & -0.260 \\
\hline 0.900 & 1183.2 & -0.285 & 1176.3 & -0.293 & 1169.3 & -0.300 & 1162.3 & -0.307 & 1155.3 & -0.313 \\
\hline 0.850 & 1144.4 & -0.382 & 1137.6 & -0.393 & 1130.7 & -0.403 & 1123.9 & -0.413 & 1117.0 & -0.424 \\
\hline 0.800 & 1109.0 & -0.487 & 1102.3 & -0.500 & 1095.6 & -0.512 & 1088.9 & -0.524 & 1082.1 & -0.536 \\
\hline 0.750 & 1075.1 & -0.567 & 1068.5 & -0.581 & 1062.0 & -0.596 & 1055.3 & -0.610 & 1048.7 & -0.625 \\
\hline 0.690 & 1037.0 & -0.662 & 1030.6 & -0.678 & 1024.2 & -0.695 & 1017.7 & -0.711 & 1011.2 & -0.726 \\
\hline 0.650 & 1013.1 & -0.693 & 1006.8 & -0.711 & 1000.5 & -0.727 & 994.1 & -0.744 & 987.6 & -0.758 \\
\hline 0.600 & 984.7 & -0.741 & 978.5 & -0.758 & 972.3 & -0.775 & 966.0 & -0.793 & 959.7 & -0.811 \\
\hline 0.550 & 957.8 & -0.755 & 951.7 & -0.775 & 945.6 & -0.791 & 939.4 & -0.806 & 933.2 & -0.825 \\
\hline 0.500 & 932.3 & -0.779 & 926.3 & -0.798 & 920.3 & -0.812 & 914.2 & -0.827 & 908.1 & -0.845 \\
\hline 0.450 & 908.1 & -0.778 & 902.2 & -0.794 & 896.2 & -0.810 & 890.2 & -0.827 & 884.2 & -0.844 \\
\hline 0.400 & 885.0 & -0.761 & 879.2 & -0.777 & 873.3 & -0.793 & 867.4 & -0.808 & 861.5 & -0.823 \\
\hline 0.350 & 863.0 & -0.729 & 857.3 & -0.748 & 851.5 & -0.762 & 845.7 & -0.774 & 839.9 & -0.788 \\
\hline 0.300 & 842.1 & -0.679 & 836.4 & -0.694 & 830.8 & -0.707 & 825.1 & -0.723 & 819.3 & -0.735 \\
\hline 0.250 & 822.1 & -0.627 & 816.6 & -0.639 & 811.0 & -0.649 & 805.4 & -0.659 & 799.7 & -0.672 \\
\hline 0.200 & 802.9 & -0.528 & 797.4 & -0.538 & 791.9 & -0.547 & 786.4 & -0.557 & 780.8 & -0.568 \\
\hline 0.150 & 784.5 & -0.414 & 779.1 & -0.421 & 773.7 & -0.427 & 768.2 & -0.435 & 762.7 & -0.444 \\
\hline 0.100 & 767.0 & -0.297 & 761.7 & -0.307 & 756.3 & -0.312 & 751.0 & -0.315 & 745.5 & -0.321 \\
\hline 0.080 & 760.1 & -0.237 & 754.8 & -0.244 & 749.5 & -0.247 & 744.2 & -0.250 & 738.8 & -0.254 \\
\hline 0.060 & 753.4 & -0.177 & 748.1 & -0.182 & 742.9 & -0.188 & 737.6 & -0.190 & 732.2 & -0.199 \\
\hline 0.040 & 746.7 & -0.100 & 741.5 & -0.106 & 736.2 & -0.106 & 731.0 & -0.107 & 725.6 & -0.109 \\
\hline 0.020 & 740.2 & -0.042 & 735.0 & -0.046 & 729.8 & -0.047 & 724.6 & -0.046 & 719.3 & -0.047 \\
\hline 0.000 & 733.9 & 0.000 & 728.8 & 0.000 & 723.6 & 0.000 & 718.4 & 0.000 & 713.1 & 0.000 \\
\hline
\end{tabular}


Table 4 (continued)

\begin{tabular}{|c|c|c|c|c|c|c|c|c|c|c|c|c|}
\hline \multirow{2}{*}{$x_{x_{1}}^{T / K}$} & \multicolumn{2}{|c|}{308.15} & \multicolumn{2}{|c|}{313.15} & \multicolumn{2}{|c|}{318.15} & \multicolumn{2}{|c|}{323.15} & \multicolumn{2}{|c|}{328.15} & \multicolumn{2}{|c|}{333.15} \\
\hline & $\rho / \mathrm{kg} \mathrm{m}^{-3}$ & $v^{E} / \mathrm{cm}^{3} \cdot \mathrm{mol}^{-1}$ & $\rho / \mathrm{kg}^{-\mathrm{m}^{-3}}$ & $v^{E} / \mathrm{cm}^{3} \cdot \mathrm{mol}^{-1}$ & $\rho / \mathrm{kg} \mathrm{m}^{-3}$ & $v^{E} / \mathrm{cm}^{3} \cdot \mathrm{mol}^{-1}$ & $\rho / \mathrm{kg} \cdot \mathrm{m}^{-3}$ & $v^{E} / \mathrm{cm}^{3} \cdot \mathrm{mol}^{-1}$ & $\rho / \mathbf{k g} \cdot \mathbf{m}^{-3}$ & $v^{E} / \mathrm{cm}^{3} \cdot \mathrm{mol}^{-1}$ & $\rho / \mathrm{kg}^{-\mathrm{m}^{-3}}$ & $v^{E} / \mathrm{cm}^{3} \cdot \mathrm{mol}^{-1}$ \\
\hline 1.000 & 1231.0 & 0.000 & 1223.6 & 0.000 & 1216.2 & 0.000 & 1208.7 & 0.000 & 1201.2 & 0.000 & 1193.7 & 0.000 \\
\hline 0.980 & 1213.5 & -0.091 & 1206.2 & -0.093 & 1198.9 & -0.094 & 1191.5 & -0.097 & 1184.0 & -0.099 & 1176.5 & -0.103 \\
\hline 0.960 & 1196.8 & -0.154 & 1189.5 & -0.158 & 1182.2 & -0.163 & 1174.9 & -0.167 & 1167.5 & -0.170 & 1160.0 & -0.176 \\
\hline 0.940 & 1180.1 & -0.210 & 1172.9 & -0.215 & 1165.7 & -0.221 & 1158.4 & -0.227 & 1151.0 & -0.234 & 1143.7 & -0.241 \\
\hline 0.920 & 1163.9 & -0.267 & 1156.8 & -0.274 & 1149.6 & -0.282 & 1142.4 & -0.290 & 1135.1 & -0.297 & 1127.8 & -0.307 \\
\hline 0.900 & 1148.2 & -0.321 & 1141.1 & -0.331 & 1134.0 & -0.339 & 1126.8 & -0.350 & 1119.6 & -0.360 & 1112.3 & -0.371 \\
\hline 0.850 & 1110.1 & -0.435 & 1103.1 & -0.448 & 1096.1 & -0.461 & 1089.1 & -0.474 & 1082.0 & -0.487 & 1074.8 & -0.502 \\
\hline 0.800 & 1075.3 & -0.551 & 1068.5 & -0.566 & 1061.6 & -0.582 & 1054.7 & -0.599 & 1047.7 & -0.615 & 1040.7 & -0.634 \\
\hline 0.750 & 1042.0 & -0.640 & 1035.3 & -0.656 & 1028.5 & -0.673 & 1021.7 & -0.693 & 1014.8 & -0.712 & 1007.9 & -0.734 \\
\hline 0.690 & 1004.7 & -0.744 & 998.1 & -0.764 & 991.4 & -0.784 & 984.8 & -0.805 & 978.0 & -0.827 & 971.3 & -0.851 \\
\hline 0.650 & 981.2 & -0.777 & 974.7 & -0.798 & 968.1 & -0.819 & 961.5 & -0.841 & 954.9 & -0.865 & 948.2 & -0.889 \\
\hline 0.600 & 953.3 & -0.830 & 947.0 & -0.851 & 940.5 & -0.872 & 934.0 & -0.895 & 927.5 & -0.921 & 920.9 & -0.945 \\
\hline 0.550 & 926.9 & -0.845 & 920.6 & -0.866 & 914.3 & -0.889 & 907.9 & -0.912 & 901.5 & -0.935 & 895.0 & -0.959 \\
\hline 0.500 & 901.9 & -0.865 & 895.7 & -0.886 & 889.5 & -0.908 & 883.2 & -0.930 & 876.8 & -0.955 & 870.4 & -0.980 \\
\hline 0.450 & 878.1 & -0.863 & 872.0 & -0.884 & 865.9 & -0.905 & 859.7 & -0.928 & 853.4 & -0.951 & 847.1 & -0.974 \\
\hline 0.400 & 855.5 & -0.842 & 849.5 & -0.861 & 843.5 & -0.881 & 837.4 & -0.901 & 831.2 & -0.924 & 825.0 & -0.945 \\
\hline 0.350 & 834.0 & -0.805 & 828.1 & -0.824 & 822.1 & -0.842 & 816.1 & -0.861 & 810.0 & -0.881 & 803.8 & -0.900 \\
\hline 0.300 & 813.5 & -0.751 & 807.7 & -0.768 & 801.8 & -0.784 & 795.9 & -0.801 & 789.9 & -0.820 & 783.8 & -0.839 \\
\hline 0.250 & 794.0 & -0.686 & 788.2 & -0.700 & 782.4 & -0.715 & 776.6 & -0.733 & 770.7 & -0.749 & 764.7 & -0.765 \\
\hline 0.200 & 775.2 & -0.580 & 769.5 & -0.591 & 763.8 & -0.603 & 758.0 & -0.617 & 752.2 & -0.631 & 746.3 & -0.643 \\
\hline 0.150 & 757.2 & -0.459 & 751.6 & -0.464 & 746.0 & -0.474 & 740.3 & -0.485 & 734.6 & -0.495 & 728.8 & -0.505 \\
\hline 0.100 & 740.1 & -0.328 & 734.6 & -0.336 & 729.0 & -0.343 & 723.4 & -0.351 & 717.8 & -0.357 & 712.0 & -0.362 \\
\hline 0.080 & 733.4 & -0.259 & 727.9 & -0.267 & 722.4 & -0.273 & 716.8 & -0.278 & 711.2 & -0.284 & 705.5 & -0.288 \\
\hline 0.060 & 726.8 & -0.196 & 721.4 & -0.200 & 715.9 & -0.205 & 710.3 & -0.209 & 704.7 & -0.214 & 699.1 & -0.215 \\
\hline 0.040 & 720.3 & -0.113 & 714.9 & -0.116 & 709.4 & -0.121 & 703.9 & -0.124 & 698.3 & -0.127 & 692.7 & -0.126 \\
\hline 0.020 & 714.0 & -0.050 & 708.6 & -0.051 & 703.2 & -0.055 & 697.7 & -0.056 & 692.1 & -0.057 & 686.5 & -0.057 \\
\hline 0.000 & 707.8 & 0.000 & 702.5 & 0.000 & 697.1 & 0.000 & 691.6 & 0.000 & 686.1 & 0.000 & 680.6 & 0.000 \\
\hline
\end{tabular}

Standard uncertainty $\mathrm{u}(p)=0.1 \mathrm{kPa}, \mathrm{u}(T)=0.01 \mathrm{~K}, \mathrm{u}\left(x_{1}\right)=0.002$

Expanded uncertainties $(\mathrm{k}=2) \mathrm{U}(\rho)=1.7 \mathrm{~kg} \cdot \mathrm{m}^{-3}, \mathrm{U}\left(\mathrm{v}^{E}\right)=0.011 \mathrm{~cm}^{3} \cdot \mathrm{mol}^{-1}$ 
Table 5: DCE (1) + DEE (2) binary mixture densities $\rho$ and excess molar volumes $v^{E}$ as a function of mole fraction in DCE $x_{1}$ at different temperatures $T$ and atmospheric pressure $(p=101.33 \mathrm{kPa})$..

\begin{tabular}{|c|c|c|c|c|c|c|c|c|c|c|c|c|}
\hline \multirow{2}{*}{$\begin{array}{c}T / \mathbf{K} \\
x_{1}\end{array}$} & \multicolumn{2}{|c|}{283.15} & \multicolumn{2}{|c|}{288.15} & \multicolumn{2}{|c|}{293.15} & \multicolumn{2}{|c|}{298.15} & \multicolumn{2}{|c|}{303.15} & \multicolumn{2}{|c|}{308.15} \\
\hline & $\rho / \mathbf{k g} \cdot \mathbf{m}^{-3}$ & $v^{E} / \mathrm{cm}^{3} \cdot \mathrm{mol}^{-1}$ & $\rho / \mathbf{k g} \cdot \mathbf{m}^{-3}$ & $v^{E} / \mathrm{cm}^{3} \cdot \mathrm{mol}^{-1}$ & $\rho / \mathrm{kg}^{-\mathrm{m}^{-3}}$ & $v^{E} / \mathrm{cm}^{3} \cdot \mathrm{mol}^{-1}$ & $\rho / \mathbf{k g} \mathbf{m}^{-3}$ & $v^{E} / \mathrm{cm}^{3} \cdot \mathrm{mol}^{-1}$ & $\rho / \mathbf{k g}^{-\mathrm{m}^{-3}}$ & $v^{E} / \mathrm{cm}^{3} \cdot \mathrm{mol}^{-1}$ & $\rho / \mathbf{k g}^{-\mathrm{m}^{-3}}$ & $v^{E} / \mathrm{cm}^{3} \cdot \mathrm{mol}^{-1}$ \\
\hline 1.000 & 1267.4 & 0.000 & 1260.2 & 0.000 & 1252.9 & 0.000 & 1245.6 & 0.000 & 1238.3 & 0.000 & 1231.0 & 0.000 \\
\hline 0.980 & 1254.4 & -0.065 & 1247.2 & -0.068 & 1240.0 & -0.070 & 1232.7 & -0.073 & 1225.4 & -0.077 & 1218.1 & -0.079 \\
\hline 0.960 & 1241.2 & -0.121 & 1234.0 & -0.127 & 1226.8 & -0.133 & 1219.6 & -0.138 & 1212.4 & -0.144 & 1205.1 & -0.148 \\
\hline 0.940 & 1228.5 & -0.172 & 1221.4 & -0.179 & 1214.2 & -0.187 & 1207.0 & -0.194 & 1199.8 & -0.203 & 1192.6 & -0.212 \\
\hline 0.915 & 1211.5 & -0.217 & 1204.5 & -0.228 & 1197.3 & -0.237 & 1190.2 & -0.246 & 1183.0 & -0.258 & 1175.8 & -0.269 \\
\hline 0.898 & 1200.8 & -0.253 & 1193.8 & -0.265 & 1186.7 & -0.277 & 1179.6 & -0.291 & 1172.4 & -0.300 & 1165.2 & -0.313 \\
\hline 0.850 & 1170.5 & -0.353 & 1163.5 & -0.369 & 1156.5 & -0.385 & 1149.4 & -0.402 & 1142.4 & -0.419 & 1135.2 & -0.439 \\
\hline 0.798 & 1138.3 & -0.449 & 1131.4 & -0.468 & 1124.4 & -0.489 & 1117.5 & -0.510 & 1110.4 & -0.533 & 1103.4 & -0.557 \\
\hline 0.750 & 1109.8 & -0.545 & 1103.0 & -0.567 & 1096.2 & -0.592 & 1089.3 & -0.618 & 1082.3 & -0.645 & 1075.3 & -0.675 \\
\hline 0.699 & 1079.4 & -0.613 & 1072.7 & -0.639 & 1065.9 & -0.667 & 1059.1 & -0.696 & 1052.2 & -0.727 & 1045.3 & -0.760 \\
\hline 0.649 & 1050.7 & -0.662 & 1044.0 & -0.690 & 1037.3 & -0.720 & 1030.5 & -0.752 & 1023.7 & -0.784 & 1016.8 & -0.820 \\
\hline 0.600 & 1023.2 & -0.733 & 1016.6 & -0.763 & 1010.0 & -0.796 & 1003.3 & -0.829 & 996.5 & -0.863 & 989.7 & -0.902 \\
\hline 0.550 & 995.5 & -0.765 & 988.9 & -0.795 & 982.3 & -0.828 & 975.7 & -0.865 & 969.0 & -0.901 & 962.3 & -0.942 \\
\hline 0.500 & 968.7 & -0.813 & 962.2 & -0.844 & 955.7 & -0.877 & 949.2 & -0.914 & 942.5 & -0.952 & 935.9 & -0.994 \\
\hline 0.450 & 942.2 & -0.816 & 935.8 & -0.848 & 929.3 & -0.882 & 922.8 & -0.919 & 916.3 & -0.957 & 909.7 & -0.998 \\
\hline 0.400 & 916.2 & -0.816 & 909.9 & -0.847 & 903.5 & -0.880 & 897.1 & -0.923 & 890.6 & -0.954 & 884.1 & -0.996 \\
\hline 0.350 & 890.7 & -0.783 & 884.5 & -0.813 & 878.2 & -0.846 & 871.8 & -0.881 & 865.4 & -0.917 & 858.9 & -0.958 \\
\hline 0.300 & 865.7 & -0.741 & 859.6 & -0.768 & 853.3 & -0.799 & 847.0 & -0.834 & 840.7 & -0.865 & 834.3 & -0.905 \\
\hline 0.250 & 841.3 & -0.688 & 835.2 & -0.712 & 829.0 & -0.741 & 822.9 & -0.782 & 816.5 & -0.801 & 810.2 & -0.837 \\
\hline 0.200 & 817.2 & -0.598 & 811.1 & -0.618 & 805.0 & -0.644 & 798.9 & -0.671 & 792.7 & -0.698 & 786.4 & -0.730 \\
\hline 0.150 & 793.5 & -0.484 & 787.5 & -0.499 & 781.5 & -0.520 & 775.4 & -0.544 & 769.3 & -0.567 & 763.1 & -0.595 \\
\hline 0.100 & 770.2 & -0.344 & 764.4 & -0.358 & 758.4 & -0.374 & 752.4 & -0.391 & 746.3 & -0.406 & 740.2 & -0.425 \\
\hline 0.080 & 761.1 & -0.288 & 755.3 & -0.297 & 749.4 & -0.310 & 743.5 & -0.339 & 737.4 & -0.338 & 731.2 & -0.354 \\
\hline 0.065 & 754.3 & -0.237 & 748.5 & -0.248 & 742.6 & -0.262 & 736.6 & -0.272 & 730.6 & -0.282 & 724.5 & -0.296 \\
\hline 0.050 & 747.5 & -0.184 & 741.7 & -0.193 & 735.8 & -0.205 & 729.9 & -0.217 & 723.9 & -0.220 & 717.8 & -0.230 \\
\hline 0.040 & 743.0 & -0.150 & 737.2 & -0.157 & 731.3 & -0.167 & 725.4 & -0.177 & 719.4 & -0.182 & 713.4 & -0.192 \\
\hline 0.020 & 734.0 & -0.069 & 728.2 & -0.071 & 722.4 & -0.077 & 716.5 & -0.083 & 710.5 & -0.086 & 704.5 & -0.091 \\
\hline 0.000 & 725.1 & 0.000 & 719.4 & 0.000 & 713.6 & 0.000 & 707.7 & 0.000 & 701.8 & 0.000 & 695.8 & 0.000 \\
\hline
\end{tabular}

Standard uncertainty $\mathrm{u}(p)=0.1 \mathrm{kPa}, \mathrm{u}(T)=0.01 \mathrm{~K}, \mathrm{u}\left(x_{1}\right)=0.002$

Expanded uncertainties $(\mathrm{k}=2) \mathrm{U}(\rho)=1.7 \mathrm{~kg} \cdot \mathrm{m}^{-3}, \mathrm{U}\left(\mathrm{v}^{E}\right)=0.011 \mathrm{~cm}^{3} \cdot \mathrm{mol}^{-1}$ 
Table 6: PFP parameters: molar volume $V_{i}$, thermal expansion coefficient $\alpha_{i}$, liquid heat capacity $C_{p i}$, isentropic compressibility $\kappa_{s i}$, isothermal compressibility $\kappa_{T i}$, and their standard deviations $u(x)$ for DCE.

\begin{tabular}{|c|c|c|c|c|c|c|}
\hline $\begin{array}{c}\text { PFP } \\
\text { Parameter }\end{array}$ & $a$ & $u(a)$ & $b$ & $u(b)$ & $c$ & $u(c)$ \\
\hline$V_{i} / \mathrm{cm}^{3} \cdot \mathrm{mol}^{-1}$ & 0.000149 & 0.000001 & 0.00429 & 0.00053 & 64.9 & 0.1 \\
\hline $10^{5} \alpha_{i} / \mathrm{K}^{-1}$ & -0.000498 & 0.000001 & 0.535 & 0.001 & 2.18 & 0.01 \\
\hline$C_{p i} / \mathrm{J} \cdot \mathrm{mol}^{-1} \cdot \mathrm{K}^{-1}$ & 0.00036 & 0.00003 & -0.140 & 0.016 & 138.0 & 2.6 \\
\hline $10^{9} \kappa_{S i} / \mathrm{Pa}^{-1}$ & 0.0000228 & 0.0000003 & -0.0093 & 0.0002 & 1.29 & 0.02 \\
\hline $10^{9} \kappa_{T \mathrm{i}} / \mathrm{Pa}^{-1}$ & 0.0000258 & 0.0000003 & -0.0090 & 0.0002 & 1.21 & 0.02 \\
\hline
\end{tabular}

Table 7: PFP parameters: molar volume $V_{i}$, thermal expansion coefficient $\alpha_{i}$, liquid heat capacity $C_{p i}$, isentropic compressibility $\kappa_{s i}$, isothermal compressibility $\kappa_{T i}$, and their standard deviations $u(x)$ for MTBE.

\begin{tabular}{ccccccc}
\hline $\begin{array}{c}\text { PFP } \\
\text { Parameter }\end{array}$ & $\boldsymbol{a}$ & $\boldsymbol{u}(\boldsymbol{a})$ & $\boldsymbol{b}$ & $\boldsymbol{u}(\boldsymbol{b})$ & $\boldsymbol{c}$ & $\boldsymbol{u}(\boldsymbol{c})$ \\
\hline $\boldsymbol{V}_{\boldsymbol{i}} / \mathbf{c m}^{\mathbf{3}} \cdot \mathbf{m o l}^{\mathbf{1}}$ & 0.000423 & 0.000003 & -0.0798 & 0.0002 & 106.1 & 0.1 \\
$\mathbf{1 0}^{\mathbf{3}} \boldsymbol{\alpha}_{\boldsymbol{i}} / \mathbf{K}^{-\mathbf{1}}$ & -0.0000122 & 0.0000001 & 0.0123 & 0.0001 & -1.14 & 0.01 \\
$\boldsymbol{C}_{\boldsymbol{p} \boldsymbol{i}} / \mathbf{J} \cdot \mathbf{m o l}^{-\mathbf{1}} \cdot \mathbf{K}^{-\mathbf{1}}$ & 0.000444 & 0.000002 & 0.040 & 0.001 & 137.98 & 0.01 \\
$\mathbf{1 0}^{\boldsymbol{9}} \boldsymbol{\kappa}_{S i} / \mathbf{P a}^{-\mathbf{1}}$ & 0.0000986 & 0.0000001 & -0.0454 & 0.0008 & 6.05 & 0.01 \\
$\mathbf{1 0}^{\boldsymbol{9}} \boldsymbol{\kappa}_{T_{\mathrm{i}}} / \mathbf{P a}^{-\mathbf{1}}$ & 0.000105 & 0.000001 & -0.0455 & 0.0007 & 5.87 & 0.01 \\
\hline
\end{tabular}

Table 8: PFP parameters: molar volume $V_{i}$, thermal expansion coefficient $\alpha_{i}$, liquid heat capacity $C_{p i}$, isentropic compressibility $\kappa_{s i}$, isothermal compressibility $\kappa_{T i}$, and their standard deviations $u(x)$ for DIPE.

\begin{tabular}{|c|c|c|c|c|c|c|}
\hline $\begin{array}{c}\text { PFP } \\
\text { Parameter }\end{array}$ & $a$ & $u(a)$ & $b$ & $u(b)$ & $c$ & $u(c)$ \\
\hline$V_{i} / \mathrm{cm}^{3} \cdot \mathrm{mol}^{-1}$ & 0.000521 & 0.000004 & -0.1032 & 0.0002 & 126.7 & 0.1 \\
\hline $10^{3} \alpha_{i} / \mathrm{K}^{-1}$ & -0.0000129 & 0.0000001 & 0.0129 & 0.0001 & -1.24 & -0.01 \\
\hline$C_{p i} / \mathrm{J} \cdot \mathrm{mol}^{-1} \cdot \mathbf{K}^{-1}$ & -0.00049 & 0.00007 & 0.70 & 0.04 & 50.23 & 0.01 \\
\hline $10^{9} \kappa_{S i} / \mathrm{Pa}^{-1}$ & 0.000121 & 0.000001 & -0.0574 & 0.0009 & 7.78 & 0.01 \\
\hline $10^{9} \kappa_{T \mathrm{i}} / \mathrm{Pa}^{-1}$ & 0.000129 & 0.000001 & -0.0582 & 0.0009 & 7.69 & 0.01 \\
\hline
\end{tabular}

Table 9: PFP parameters: molar volume $V_{i}$, thermal expansion coefficient $\alpha_{i}$, liquid heat capacity $C_{p i}$, isentropic compressibility $\kappa_{s i}$, isothermal compressibility $\kappa_{T i}$, and their standard deviations $u(x)$ for DEE.

\begin{tabular}{|c|c|c|c|c|c|c|}
\hline $\begin{array}{c}\text { PFP } \\
\text { Parameter }\end{array}$ & $a$ & $u(a)$ & $b$ & $u(b)$ & $c$ & $\boldsymbol{u}(\boldsymbol{c})$ \\
\hline$V_{i} / \mathrm{cm}^{3} \cdot \mathrm{mol}^{-1}$ & 0.00048 & 0.00003 & -0.108 & 0.001 & 94.7 & 0.2 \\
\hline $10^{3} \alpha_{i} / \mathrm{K}^{-1}$ & -0.000018 & 0.0000004 & 0.0170 & 0.0001 & -1.80 & 0.01 \\
\hline$C_{p i} / \mathrm{J} \cdot \mathrm{mol}^{-1} \cdot \mathrm{K}^{-1}$ & 0.00008 & 0.00003 & 0.14 & 0.02 & 124 & 2 \\
\hline $10^{9} \kappa_{S i} / \mathrm{Pa}^{-1}$ & 0.000118 & 0.000002 & -0.054 & 0.001 & 7.0 & 0.2 \\
\hline $10^{9} \kappa_{T \mathrm{i}} / \mathrm{Pa}^{-1}$ & 0.000132 & 0.000002 & -0.056 & 0.001 & 7.0 & 0.1 \\
\hline
\end{tabular}


Table 6: Flory parameters $\chi_{12}^{0}, d$ and $e$ for the three systems DCE + ether at temperature $T$ from 278.15 to 333.15 K.

\begin{tabular}{|c|c|c|c|c|c|c|c|c|c|}
\hline \multirow{2}{*}{$T / \mathbf{K}$} & \multicolumn{3}{|c|}{ DCE + MTBE } & \multicolumn{3}{|c|}{ DCE + DIPE } & \multicolumn{3}{|c|}{ DCE + DEE } \\
\hline & $\chi_{12}^{0} / \mathrm{J}_{\mathrm{c}} \mathrm{cm}^{-3}$ & d & $\mathbf{e}$ & $\chi_{12}^{0} / \mathrm{J}_{\mathrm{J}} \mathrm{cm}^{-3}$ & d & $\mathbf{e}$ & $\chi_{12}^{0} / J_{.} \mathrm{cm}^{-3}$ & d & e \\
\hline 278.15 & -12.399 & 0.858 & -0.661 & & & & & & \\
\hline 283.15 & -11.454 & 0.850 & -0.696 & -16.640 & 1.012 & 0.042 & -10.359 & 0.929 & -0.377 \\
\hline 288.15 & -10.501 & 0.843 & -0.723 & -15.098 & 1.014 & 0.051 & -9.020 & 0.929 & -0.377 \\
\hline 293.15 & -9.436 & 0.835 & -0.756 & -13.444 & 1.020 & 0.070 & -7.893 & 0.914 & -0.445 \\
\hline 298.15 & -8.684 & 0.822 & -0.811 & -11.795 & 1.026 & 0.092 & -7.016 & 0.894 & -0.540 \\
\hline 303.15 & -7.954 & 0.811 & -0.860 & -10.245 & 1.032 & 0.113 & -5.847 & 0.890 & -0.550 \\
\hline 308.15 & -7.175 & 0.799 & -0.906 & -8.870 & 1.039 & 0.138 & -5.128 & 0.876 & -0.615 \\
\hline 313.15 & -6.600 & 0.789 & -0.946 & -7.572 & 1.054 & 0.189 & & & \\
\hline 318.15 & -6.014 & 0.784 & -0.962 & -6.367 & 1.072 & 0.251 & & & \\
\hline 323.15 & -5.594 & 0.777 & -0.988 & -5.267 & 1.100 & 0.342 & & & \\
\hline 328.15 & & & & -4.253 & 1.137 & 0.469 & & & \\
\hline 333.15 & & & & -3.294 & 1.214 & 0.727 & & & \\
\hline
\end{tabular}

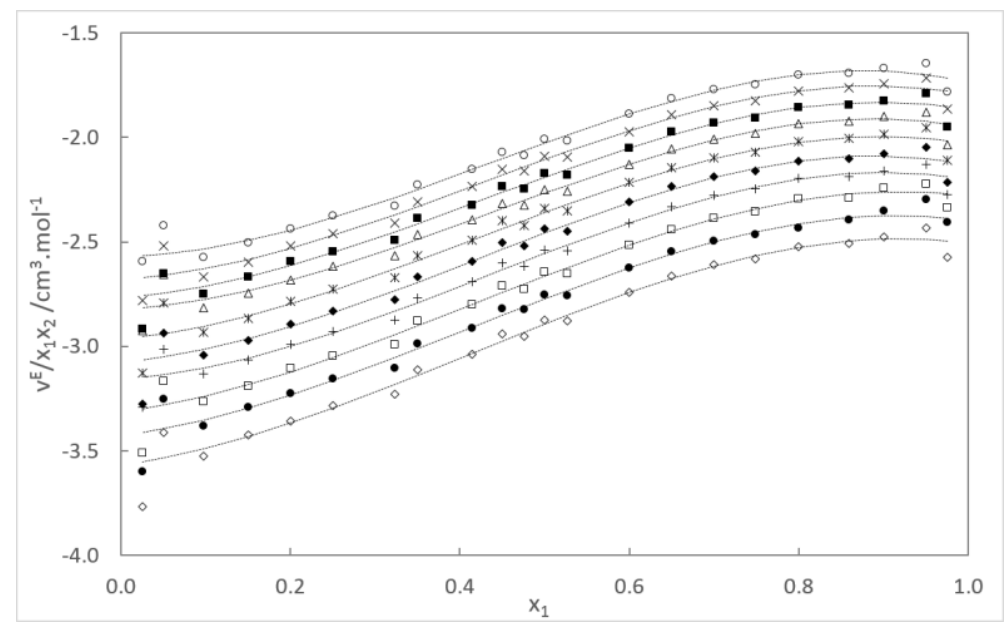

Figure 1: $v^{E} / x_{1} x_{2}$ for DCE (1) + MTBE (2) mixture according to $x_{1}$ different temperatures: $\circ: 278 \mathrm{~K}$, ×: $283 \mathrm{~K}$, 匹: $288 \mathrm{~K}, \Delta: 293 \mathrm{~K}, *: 298 \mathrm{~K}, \triangleleft: 303 \mathrm{~K},+: 308 \mathrm{~K}, \square: 313 \mathrm{~K}, \bullet: 318 \mathrm{~K}, \diamond: 323 \mathrm{~K}, \cdots:$ RedlichKister correlation. 


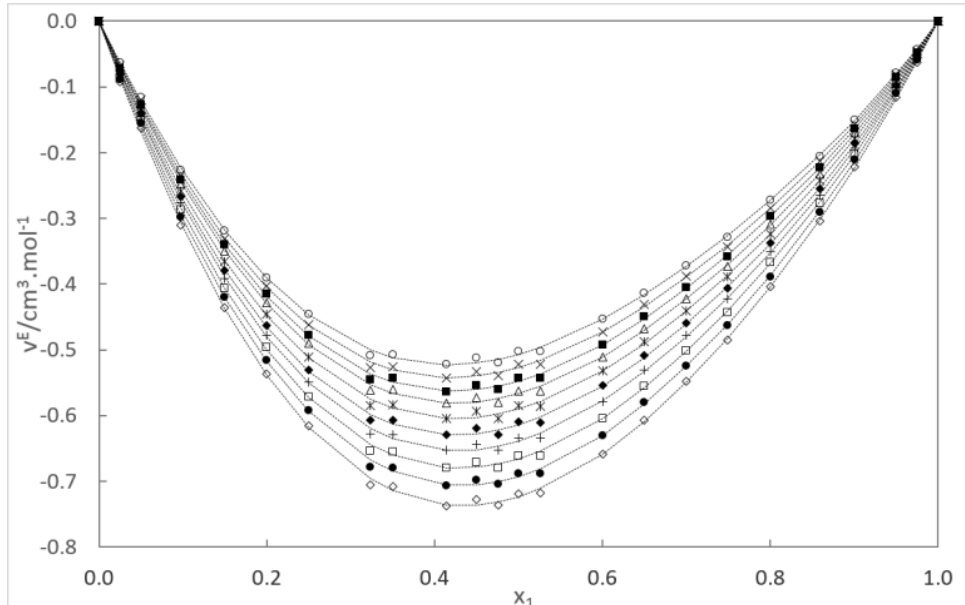

Figure 2: $v^{E}$ for DCE (1) + MTBE (2) mixture according to $x_{1}$ different temperatures: $\circ: 278 \mathrm{~K}, \times: 283 \mathrm{~K}$, п: $288 \mathrm{~K}, \Delta: 293 \mathrm{~K}, *: 298 \mathrm{~K}, \diamond: 303 \mathrm{~K},+: 308 \mathrm{~K}$, , $: 313 \mathrm{~K}, \bullet: 318 \mathrm{~K}, \diamond: 323 \mathrm{~K}, \cdots$ : Redlich-Kister correlation.

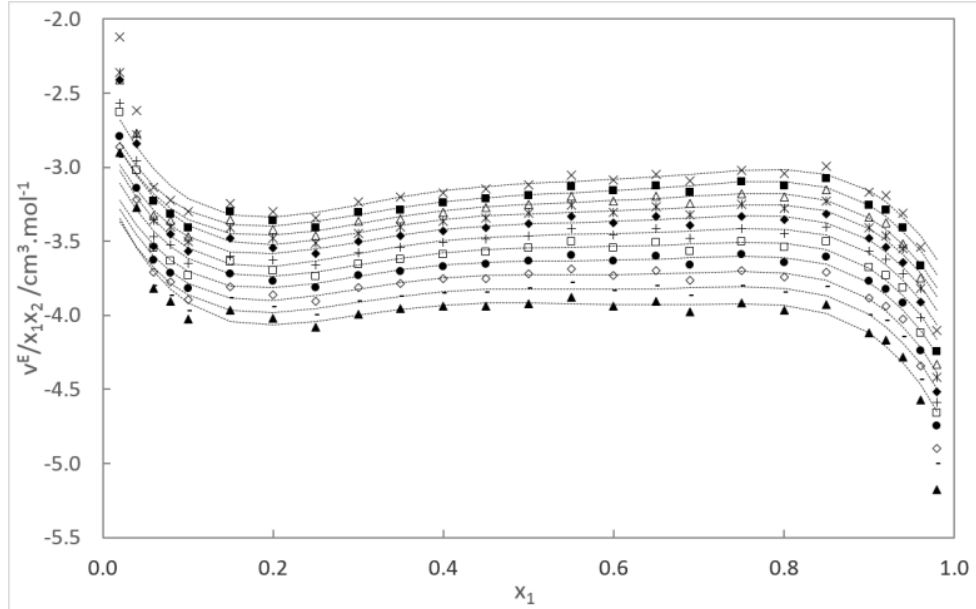

Figure 3: $v^{E} / x_{1} x_{2}$ for DCE (1) + DIPE (2) mixture according to $x_{1}$ at different temperatures: $\times: 283 \mathrm{~K}, \mathrm{m:}$ $288 \mathrm{~K}, \Delta: 293 \mathrm{~K}, *: 298 \mathrm{~K}, \triangleleft: 303 \mathrm{~K}$, t: $308 \mathrm{~K}, \square: 313 \mathrm{~K}, \bullet: 318 \mathrm{~K}, \diamond: 323 \mathrm{~K}$, -: $328 \mathrm{~K}, \Delta: 333 \mathrm{~K}, \cdots:$ Redlich-Kister correlation. 


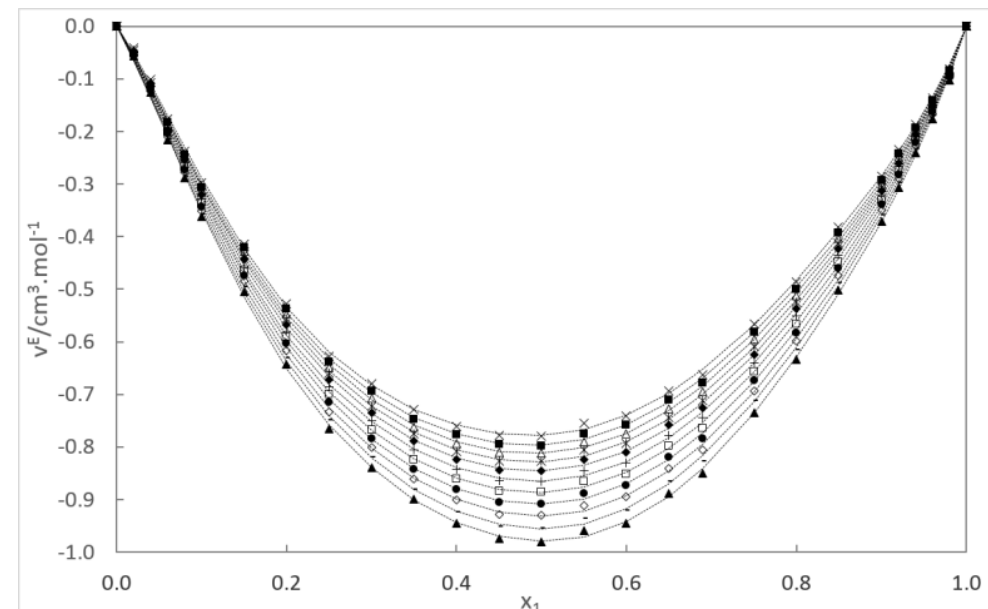

Figure 4: $v^{E}$ for DCE (1) + DIPE (2) mixture according to $x_{1}$ at different temperatures: $\times: 283 \mathrm{~K}, \mathrm{a}: 288 \mathrm{~K}$, $\Delta: 293 \mathrm{~K}, *: 298 \mathrm{~K}, \triangleleft: 303 \mathrm{~K}$, +: $308 \mathrm{~K}, \square: 313 \mathrm{~K}, \bullet: 318 \mathrm{~K}, \diamond: 323 \mathrm{~K},-: 328 \mathrm{~K}, \Delta$ : $333 \mathrm{~K}, \cdots:$ RedlichKister correlation.

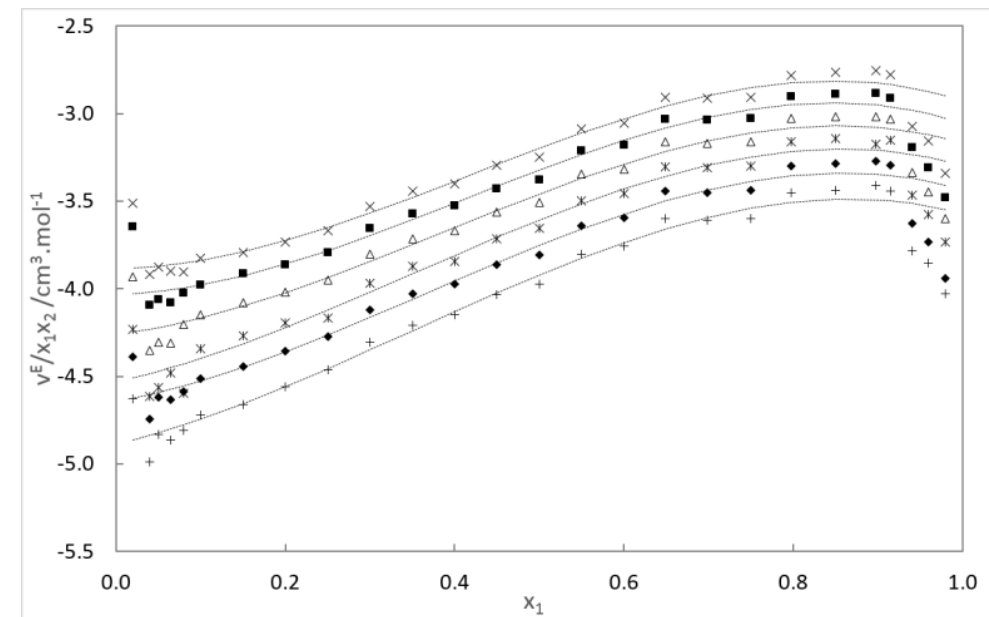

Figure 5: $v^{E} / x_{1} x_{2}$ for DCE (1) + DEE (2) mixture according to $x_{1}$ at different temperatures: $\times: 283 \mathrm{~K}$, च: $288 \mathrm{~K}, \Delta: 293 \mathrm{~K}, *$ : $298 \mathrm{~K}, \diamond: 303 \mathrm{~K},+: 308 \mathrm{~K}, \cdots$ : Redlich-Kister correlation.

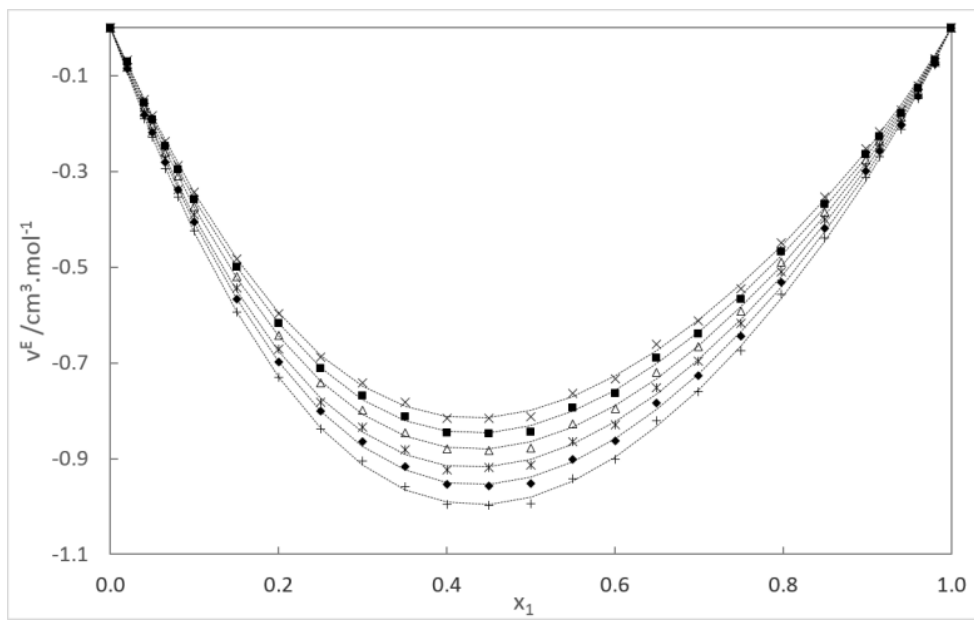

Figure 6: $v^{E}$ for DCE (1) + DEE (2) mixture according to $x_{1}$ at different temperatures: $\times: 283 \mathrm{~K}, \varpi: 288 \mathrm{~K}$, $\Delta: 293 \mathrm{~K}, *: 298 \mathrm{~K}, \triangleleft: 303 \mathrm{~K}$, +: $308 \mathrm{~K}, \cdots$ : Redlich-Kister correlation. 
To the best of our knowledge, no data from the literature, on the DCE + DEE and DCE + DIPE binary systems, was found. One study for the DCE + MTBE binary system was reported in the literature, by Sharma and al. ${ }^{17}$ at $303 \mathrm{~K}$. A comparison of the two isotherms is presented in Figure 7. We can observe a good agreement between our data and the literature data, $v^{E}$ as a function of $\mathrm{x}_{1}$ follows the same trend.

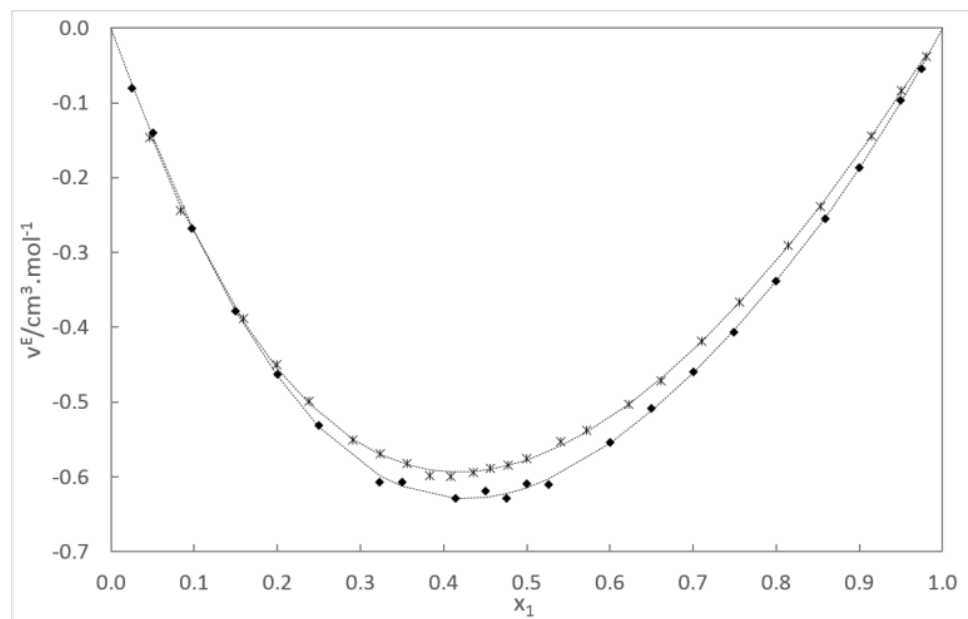

Figure 7: $v^{E}$ for DCE (1) + MTBE (2) mixture according to $x_{1}$ at $303 \mathrm{~K}$ : $\times$ : experimental data from Sharma and al. ${ }^{17}, \star$ : our experimental data, $\cdots$ : Redlich-Kister correlation.

\section{Discussion}

\section{Excess molar volume}

The excess molar volumes are negative for the three binary systems, as we can see in Figure 2 to 6 . and we can see that the amplitude increase with the temperature. The negative $\mathrm{v}^{\mathrm{E}}$ for the three systems can be interpreted by the packing effect: a good insertion of the DCE molecule in the ethers or by a dipole-dipole interaction as the dipoles moments of the compounds are closed (DCE: 1.439 D, MTBE: 1.361 D, DIPE: 1.302 D and DEE: $1.151 \mathrm{D}$, data from Simulis Thermodynamics ${ }^{\mathrm{TM}}$ from PROSIM, France). The $v^{E}$ values are symmetrical for the DCE + DEE and DCE + DIPE binary systems and slightly asymmetrical for the DCE + MTBE system. The maximal absolute $v^{E}$ (at $x=0.5$ ), over the entire temperature range, increases for the three binary systems with the temperature. At $298 \mathrm{~K}$, the maximal absolute $v^{E}$ for the DCE + MTBE, DCE + DIPE and DCE + DEE systems are respectively $0.586,0.827$ and 0.919 . This amplitude difference between the three systems is due to the steric hindrance of the ether. When the ether molecule has a lower steric hindrance, it is better packed with the DCE molecule.

Following the method of Desnoyers and Perron ${ }^{16}, v^{E} / x_{1} x_{2}$ as a function of $\mathrm{x}_{1}$ was also plotted for each binary systems, in Figure 1, Figure 3 and Figure 5. This term is directly related to the apparent molar volume following Eq. 23:

$\frac{v^{E}}{x_{1} x_{2}}=\frac{v_{\Phi_{1}}-v_{1}^{0}}{x_{1}}=\frac{v_{\Phi_{2}}-v_{2}^{0}}{x_{2}}$

These graphics don't show any particular behavior relating to the behavior of the studied systems, in the diluted areas. The utilization of a polynomial expression like the one suggested by Redlich-Kister is not well-adapted for the representation of the excess volume for our three systems in the diluted areas. In effect, a good representation of excess volume requires a lot of parameters and can lead to a bad representation of the derivative properties. It is for this reason that we have also 
considered another approach by considering the partial molar volume, obtained by derivative of the volume versus the mole number.

The apparent molar volumes of DCE in ether calculated from experimental data with Eq 11, and the partial molar volumes of DCE in ether estimated using the Redlich-Kister parameters $A_{n}$ (Eq 8 ) for the three systems as a function of the DCE molar fraction $x_{1}$ are respectively presented in Figure 8 to 10 at two temperatures.

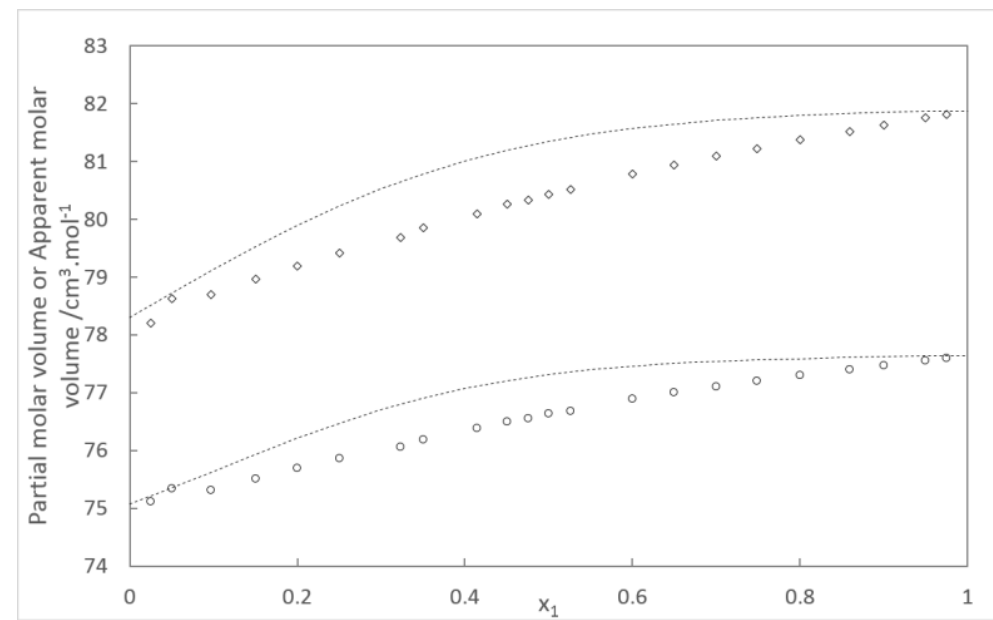

Figure 8: Apparent molar volume of DCE in the DCE + MTBE mixture according to $x_{1}$ at two temperatures: $\odot: 278 \mathrm{~K}, \diamond: 323 \mathrm{~K}, \cdots:$ Estimated partial molar volume (from Eq. 8).

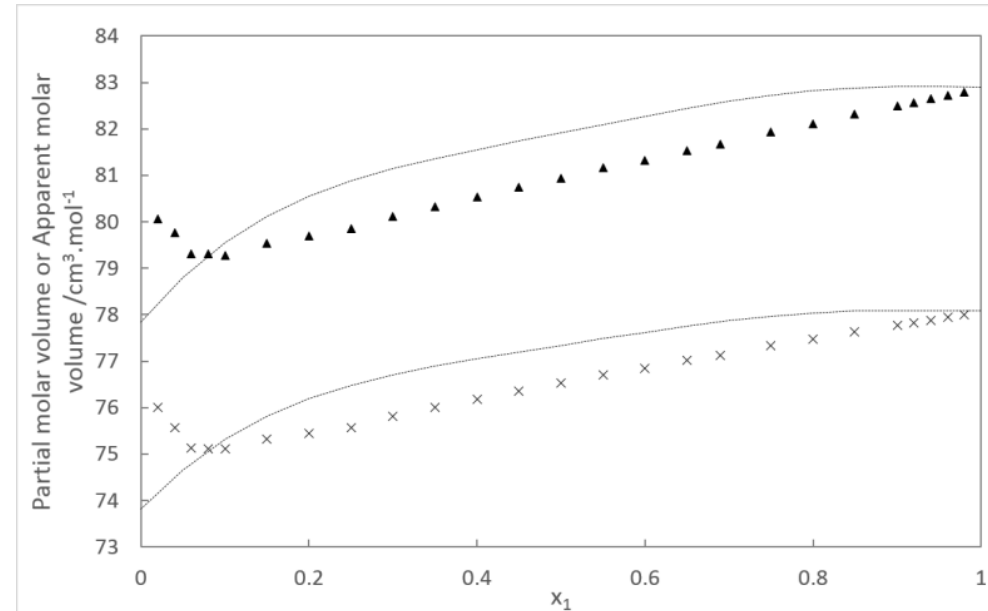

Figure 9: Apparent molar volume of DCE in the DCE + DIPE mixture according to $x_{1}$ at two temperatures: $\times$ : $283 \mathrm{~K}, \Delta: 333 \mathrm{~K}, \cdots:$ Estimated partial molar volume (from Eq. 8). 


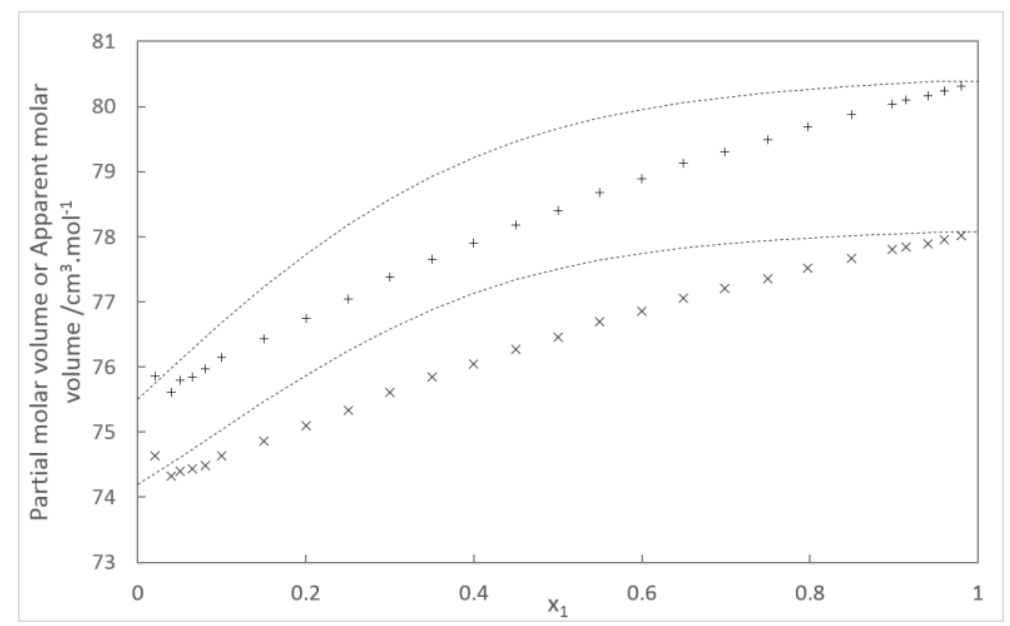

Figure 10: Apparent molar volume of DCE in the DCE + DEE mixture according to $x_{1}$ at two temperatures: $x: 283 \mathrm{~K},+: 308 \mathrm{~K}, \cdots$ : Estimated partial molar volume (from Eq. 8).

The partial molar volume corresponds to the volume occupied by the solute in the solution. At infinite dilution, the main molecular interactions in the mixture are the solute-solvent and solventsolvent interactions. It is an important property as it is linked to the nature of the molecular interaction between the solute and the solvent. As it can be seen in Figure 8 to 10, the results obtained using both approaches do not always agree, especially for the binary systems DCE + DEE and DCE + DIPE for regions with low DCE molar fraction. More information is needed in the very dilute region. Lastly, we have considered the Prigogine-Flory-Patterson (PFP) model for the data treatment.

\section{Prigogine-Flory-Patterson model}

To apply the PFP theory to our experimental data, we need to estimate several properties of the different molecules, such as the isothermal and isentropic compressibility $\kappa_{T i}$ and $\kappa_{s i}$, the molar volume $V_{i}$ and the thermal expansion coefficient $\alpha_{i}$. The experimental densities and speed of sound of the four compounds at different temperatures used to calculate the isentropic compressibility are presented in Table 2 . The experimental liquid heat capacities, $C_{p i}$, measured by Goralski and al ${ }^{18}$ are the ones used for the DCE pure compound. For the MTBE constituent, the values predicted by REFPROP $^{19}$ are used. For the DIPE molecule, we have used the data from Paramo and al. $^{20}$. And for the DEE, we have used the data from Counsell and al. ${ }^{21}$.

The thermodynamics equations presented in section 0 , are used to calculate these properties. The hypothesis that these properties can be expressed by a polynomial equation of the second-degree dependant on the temperature defined by Eq. 24, in our experimental conditions of temperature and pressure, was made.

$F(T)=a T^{2}+b T+c$

The parameters for the application of the PFP theory for the DCE, MTBE, DIPE, and DEE compound and their standard deviation, $u(x)$, are presented respectively in Table 6 to 9 .

To treat the experimental with the PFP method, the $\chi_{12}$ parameter is regressed to minimize the objective function, $F c$, defined in Eq. 25. The PFP parameters presented in Table 6 to 9 are used, according to the temperature. 
$F c=\sum_{i}\left(v_{e x p}^{E}-v_{P F P}^{E}\right)^{2}$

As suggested by Coquelet and al. ${ }^{14}$, and applied by Ali Raza et al. ${ }^{22}$ we have also considered that the $\chi_{12}$ parameter is dependent on the composition of the solution following Eq. 26.

$\chi_{12}=\chi_{12}^{0}\left(d+e\left(\varphi_{1}-\varphi_{2}\right)\right)$

where the Flory parameter $\chi_{12}^{0}$ is estimated independently of the composition.

The PFP model is used on the experimental data for the three binary systems and at all the temperatures. The $\chi_{12}^{0}$, d and e parameters (from Eq. 26) estimated with the objective function, $\mathrm{Fc}$, are presented in Table 10. To compare the dependence or independence of the Flory parameter to the composition, the two PFP treatments for the three binary systems, respectively DCE + MTBE, DCE + DIPE, and DCE + DEE, at the lowest and the highest temperature of the experimental conditions, are presented in Figure 11 to 13. Consequently, we can see that the PFP model with a Flory parameter dependent on the composition represents better the excess molar volume estimated from the experimental data, especially for the DCE + MTBE mixture.
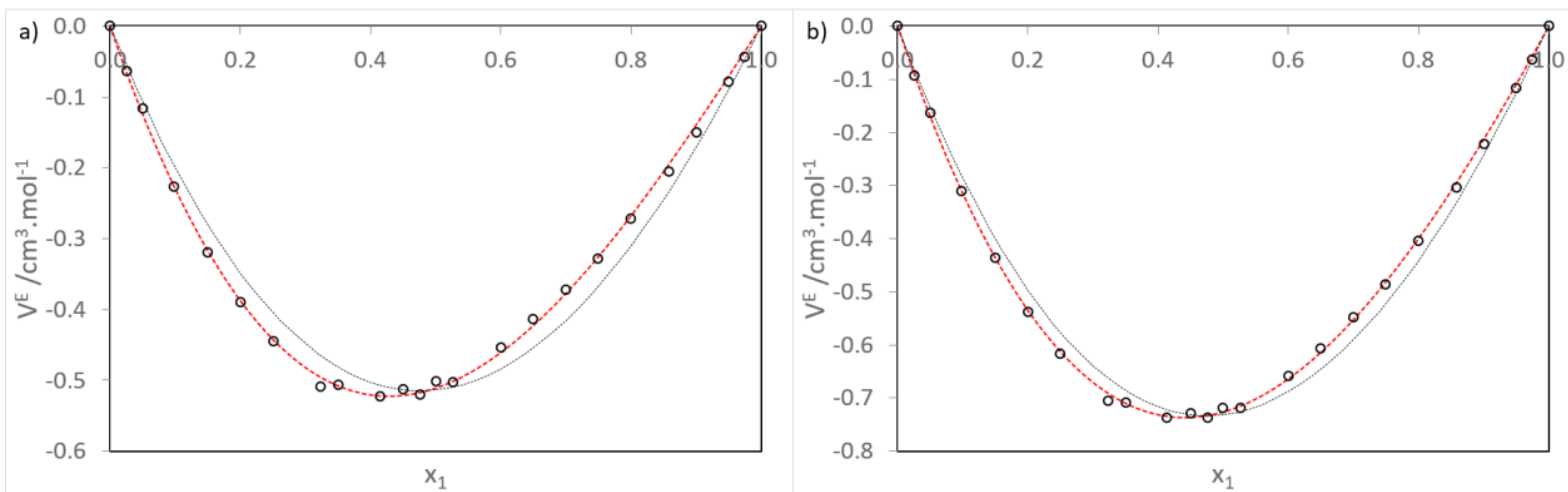

Figure 11: $v^{E}$ for DCE (1) + MTBE (2) system according to $x_{1} \cdots$ : PFP model with $\chi_{12}$ parameter independent of the composition and $\cdots$ : PFP model with $\chi_{12}$ parameter dependent of the composition; a) $T$ $=278 \mathrm{~K}$ and $\mathrm{b}) \mathrm{T}=323 \mathrm{~K}$.
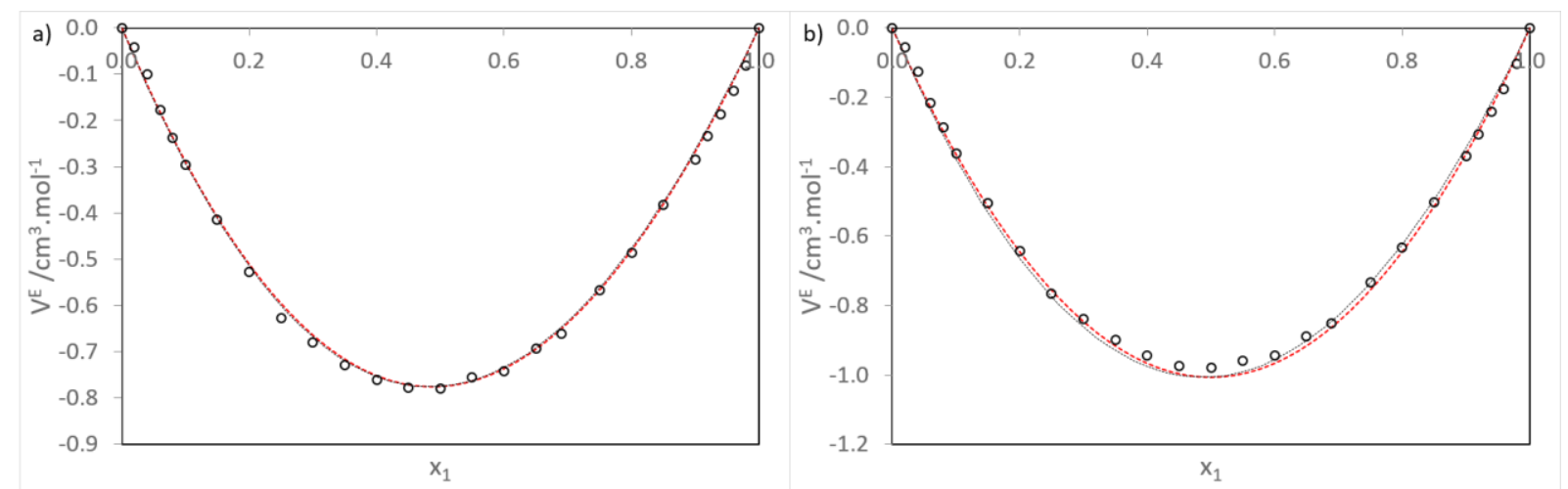

Figure 12: $v^{E}$ for DCE (1) + DIPE (2) system according to $x_{1} \cdots$ : PFP model with $\chi_{12}$ parameter independent of the composition and '*: PFP model with $\chi_{12}$ parameter dependent of the composition; a) $T$ $=283 \mathrm{~K}$ and b) $T=333 \mathrm{~K}$. 

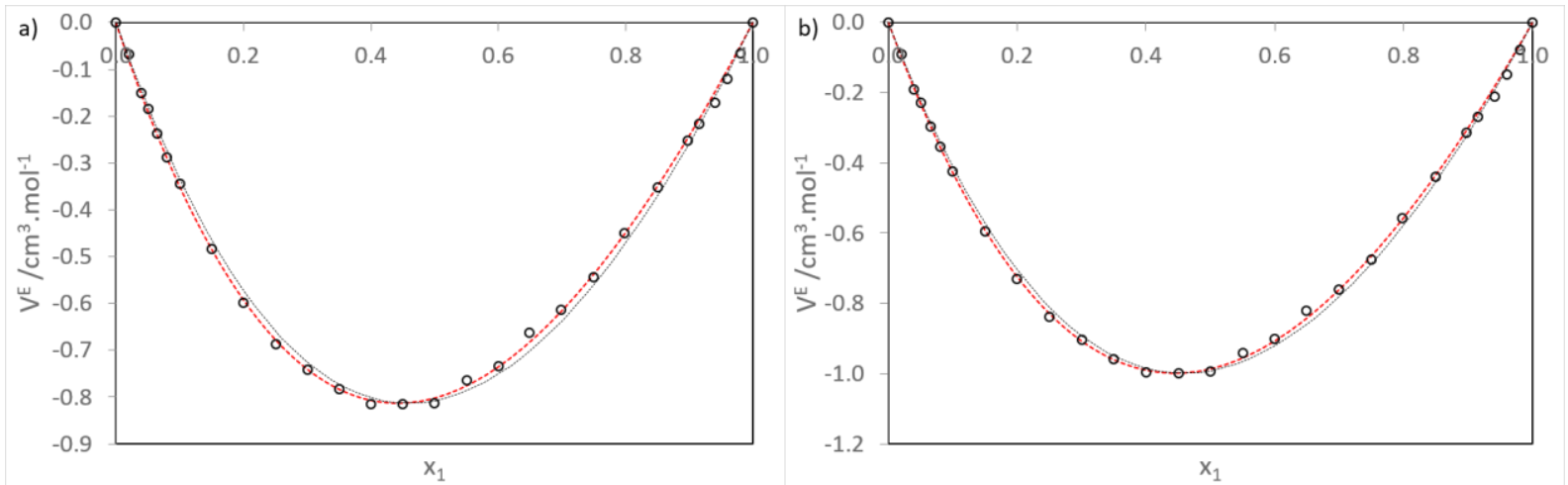

Figure 13: $v^{E}$ for DCE (1) + DEE (2) system according to $x_{1} \cdots$ : PFP model with $\chi_{12}$ parameter independent of the composition and $\cdots$ : PFP model with $\chi_{12}$ parameter dependent of the composition; a) $T=283 \mathrm{~K}$ and b) $T=308 \mathrm{~K}$.

With the PFP model, we can also analyze the weight of the three contributions $\mathrm{v}_{\text {int }}, \mathrm{v}_{\mathrm{fv}}$ and $\mathrm{v}_{\mathrm{P}}$ on the excess molar volume. In Figure 14 to 16, for three different temperatures of each binary system, the estimation with the PFP model of the three contributions according to the molar fraction of DCE is represented. For the DCE + MTBE mixture, the effect of the free volume contribution seems to slightly increase with the temperature and is less important than the other two. Moreover, the internal pressure is the main contributor to the excess molar volume, it increases when the temperature increases. The interactional contribution is also important at low temperature but it decreases when the temperature increases to the point where it has less impact than the $\mathrm{v}_{\mathrm{fv}}$. The observations are the same for the DCE + DIPE binary system. For the DCE + DEE binary mixture, the observations are also similar, nevertheless, the interactional contribution is no more significant, as the free volume contribution, at low temperatures. To the best of our knowledge, the only PFP treatment of any of the binary systems studied was the one on the DCE + MTBE binary system reported by Sharma and al. ${ }^{17}$ at $303 \mathrm{~K}$. They also found, like us, that the $P^{*}$ interaction is the main contribution to the excess molar volume of the mixture. 

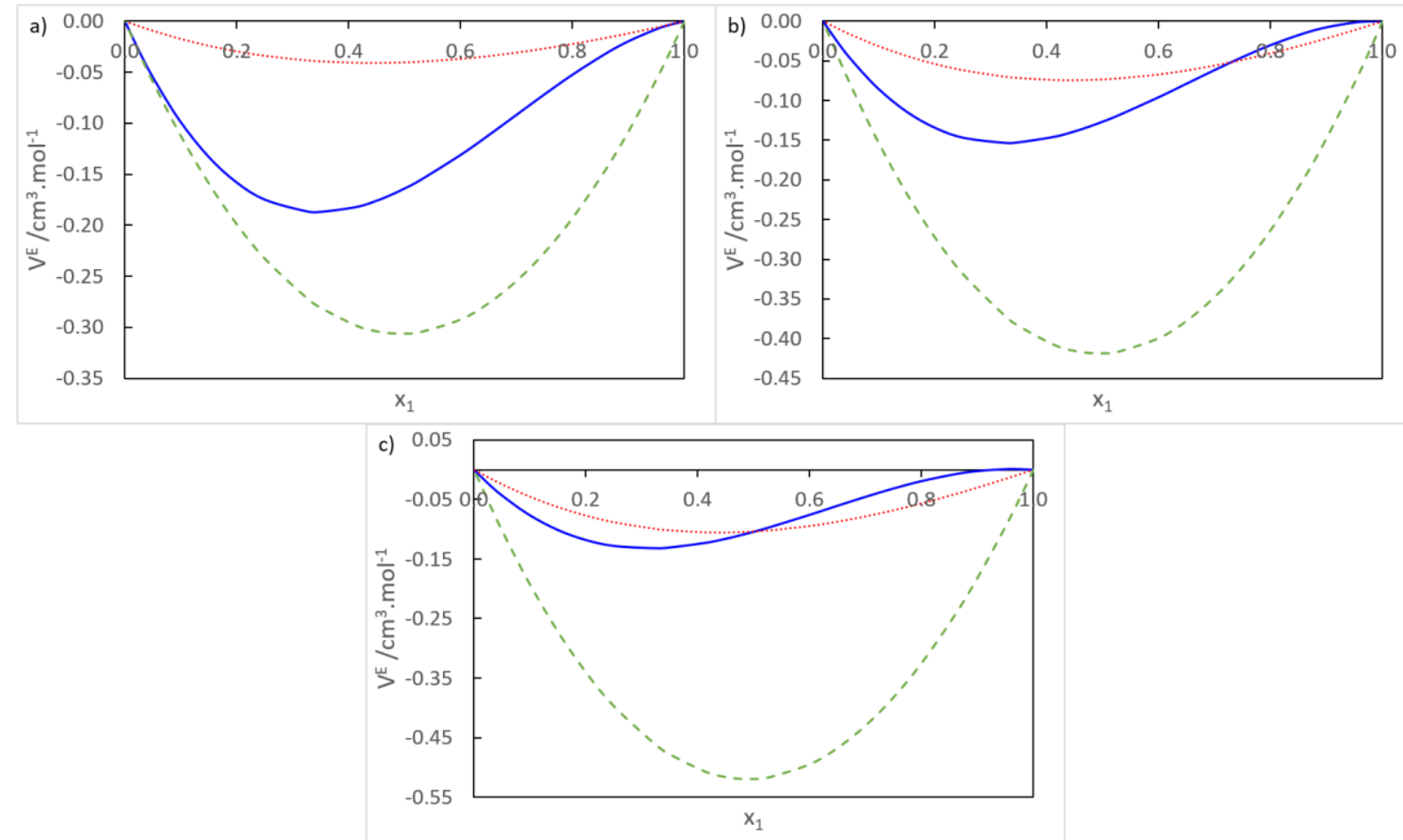

Figure 14: PFP contributions to $v^{E}$ for DCE (1) + MTBE (2) system according to $x_{1} ;-: v_{i n t}, \cdots: v_{f v},--: v_{P *}$; a) $T=278 \mathrm{~K}$, b) $T=308 \mathrm{~K}$ and c) $T=323 \mathrm{~K}$.
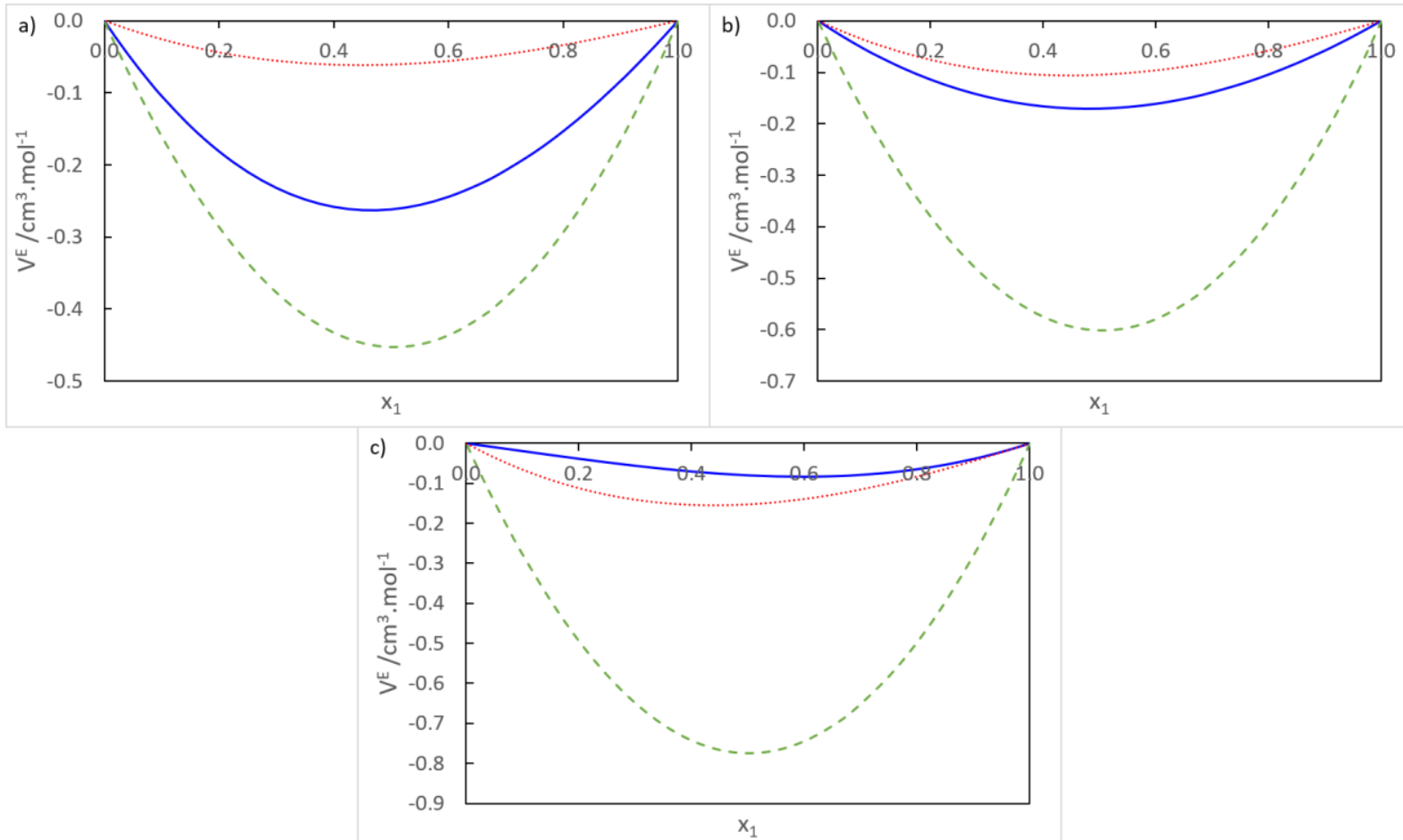

Figure 15: PFP contributions to $v^{E}$ for DCE (1) + DIPE (2) system according to $x_{1} ;$ - $v_{i n t}, \cdots: v_{f v},--: v_{P *}$; a) $T=283 \mathrm{~K}$, b) $T=308 \mathrm{~K}$ and c) $T=333 \mathrm{~K}$. 


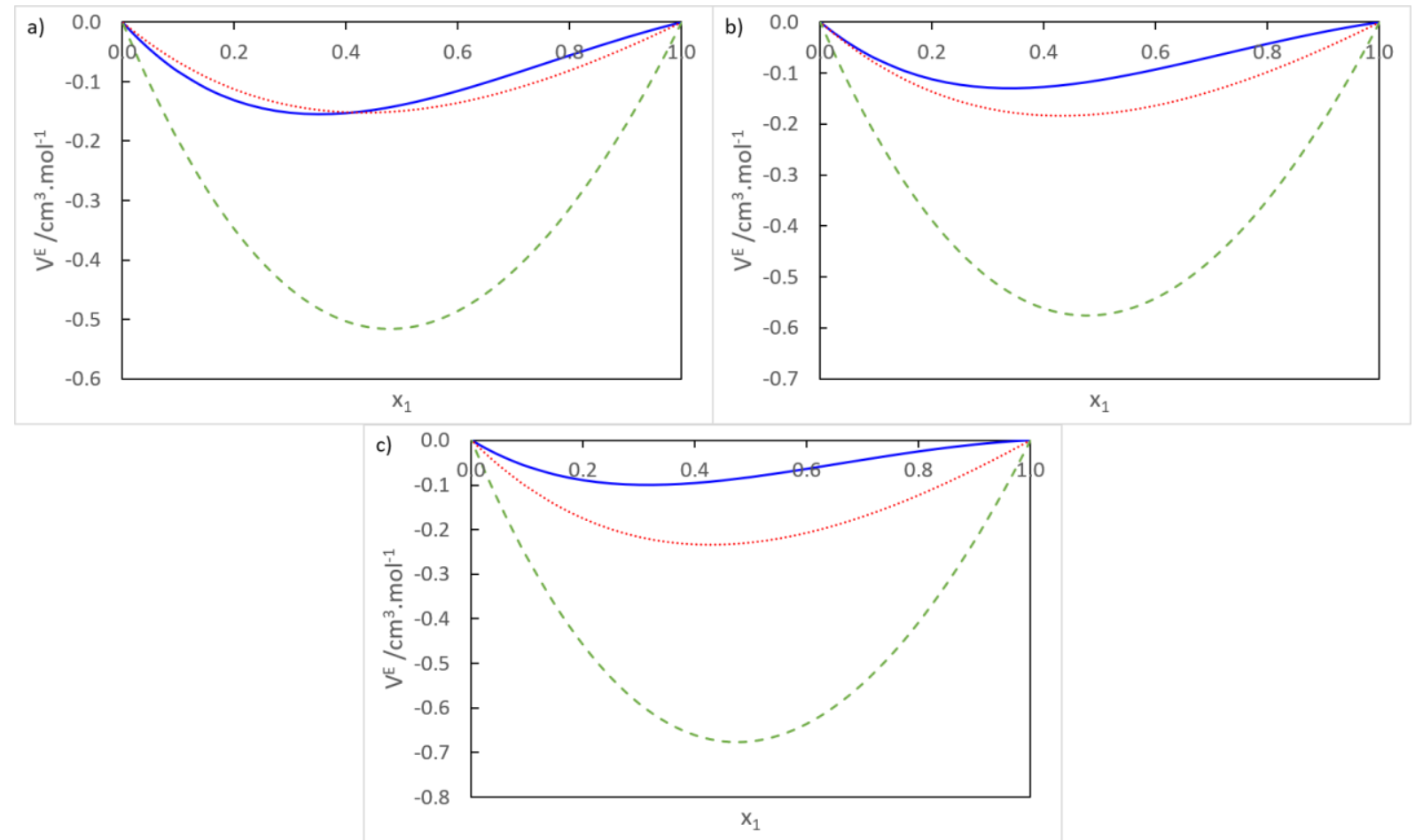

Figure 16: PFP contributions to $v^{E}$ for DCE (1) + DEE (2) system according to $x_{1} ;-: v_{i n t}, \cdots: v_{f v},--: v_{P *}$; a) $T$ $=283 \mathrm{~K}$, b) $T=293 \mathrm{~K}$ and c) $T=308 \mathrm{~K}$.

\section{Conclusion}

The densities of the DCE + MTBE, DCE + DIPE, and DCE + DEE binary solutions were determined at atmospheric pressure and temperature $T=(278.15$ to 333.15$) \mathrm{K}$. The pure compound densities and speed of sound were also measured with a vibrating tube density meter. Furthermore, the excess molar volumes of these mixtures were estimated and correlated using the Redlich-Kister correlation. The excess molar volumes of the three binary systems are negatives for all the systems and in all the temperature range. The excess molar volume increases when the temperature increases for all the binary mixtures. We notice that the maximal absolute $v^{E}$ for the three systems increases following: DCE + MTBE < DCE + DIPE < DCE + DEE. Accordingly, this amplitude difference between the three systems is due to the steric hindrance of the different ethers. The partial volume data treatment was also applied. The PFP theory was used as well on the three systems using a $\chi_{12}$ parameter. When this Flory parameter is dependent on the composition of the solution, a better representation of the experimental results for the DCE + MTBE mixture is found. According to the PFP model, the most relevant contribution for all these mixtures is the internal pressure and reduced volume, $P^{*}$ contribution.

\section{Supporting Information Available:}

Evaluation of pure component densities, comparison with DIPPR correlation ${ }^{23}$ and literature data ${ }^{24-40}$

\section{References}

(1) Mehlman, M. A. Ethers; American Cancer Society, 2012.

(2) Chapter 11 - Process Design. In Lees' Loss Prevention in the Process Industries (Fourth Edition); Mannan, S., Ed.; Butterworth-Heinemann: Oxford, 2012; 443-508. 
(3) Valtz, A.; Coquelet, C.; Boukais-Belaribi, G.; Dahmani, A.; Belaribi, F. B. Volumetric Properties of Binary Mixtures of 1,2-Dichloroethane with Polyethers from (283.15 to 333.15) K and at Atmospheric Pressure. J. Chem. Eng. Data 2011, 56, 1629-1657.

(4) Amireche-Ziar, F.; Richon, D.; Belaribi, F. B. Excess Molar Enthalpies for Binary Mixtures of 1,2-Dichloroethane with Ethers at 298.15K and Atmospheric Pressure. Fluid Phase Equilibria 2013, 337, 255-258.

(5) Amireche-Ziar, F.; Boukais-Belaribi, G.; Jakob, A.; Mokbel, I.; Belaribi, F. B. Isothermal Vapour-Liquid Equilibria of Binary Systems of 1,2-Dichloroethane with Ethers. Fluid Phase Equilibria 2008, 268, 39-44.

(6) Benabida, H.; Coquelet, C.; Belaribi, F. B. Densities and Excess Molar Volumes of the Ternary System (1,4-Dioxane + 2-Propanol + 1,1,2,2-Tetrachloroethane) at $\mathrm{T}=288.15-318.15 \mathrm{~K}$ and at Atmospheric Pressure: Experimental Measurements and Prigogine-Flory-Patterson Modeling. J. Chem. Eng. Data 2019, 64, 5122-5131.

(7) Redlich, O.; Kister, A. T. Algebraic Representation of Thermodynamic Properties and the Classification of Solutions. Ind. Eng. Chem. 1948, 40, 345-348.

(8) Patterson, D.; Delmas, G. Corresponding States Theories and Liquid Models. Discuss. Faraday Soc. 1970, 49, 98.

(9) Flory, P. J. Statistical Thermodynamics of Liquid Mixtures. J. Am. Chem. Soc. 1965, 87, 18331838

(10) Abe, A.; Flory, P. J. The Thermodynamic Properties of Mixtures of Small, Nonpolar Molecules. J. Am. Chem. Soc. 1965, 87, 1838-1846.

(11) Prigogine, I. Molecular Theory of Solutions, North Holland Publishing.; North-Holland Pub. Co.,; New York: Amsterdam, 1957.

(12) Hansen, C. M. Hansen Solubility Parameters: A User's Handbook, Second Edition, 2nd ed.; CRC Press: Boca Raton, 2007.

(13) Afzal, W.; Valtz, A.; Coquelet, C.; Richon, D. Volumetric Properties of (Piperidine+water) Binary System: Measurements and Modeling. J. Chem. Thermodyn. 2008, 40, 47-53.

(14) Coquelet, C.; Auger, E.; Valtz, A. Density and Excess Volume for Four Systems Involving Eugenol and Furan. J. Solut. Chem. 2019, 48, 455-488.

(15) Chirico, R. D.; Frenkel, M.; Magee, J. W.; Diky, V.; Muzny, C. D.; Kazakov, A. F.; Kroenlein, K.; Abdulagatov, I.; Hardin, G. R.; Acree Jr, W. E. Improvement of Quality in Publication of Experimental Thermophysical Property Data: Challenges, Assessment Tools, Global Implementation, and Online Support. J. Chem. Eng. Data 2013, 58, 2699-2716.

(16) Desnoyers, J. E.; Perron, G. Treatment of Excess Thermodynamic Quantities for Liquid Mixtures. J. Solut. Chem. 1997, 26, 749-755.

(17) Sharma, S. C.; Joshi, I. M.; Singh, J.; Gupta, A. Excess Molar Volumes of Methyl Tert-Butyl Ether with Chloroalkanes at $30^{\circ} \mathrm{C}$. J. Solut. Chem. 1992, 21, 8.

(18) Góralski, P.; Tkaczyk, M.; Chorążewski, M. Heat Capacities of $\alpha, \omega$-Dichloroalkanes at Temperatures from $284.15 \mathrm{~K}$ to $353.15 \mathrm{~K}$ and a Group Additivity Analysis. J. Chem. Eng. Data 2003, 48, 492-496.

(19) Lemmon, E. W.; Huber, M. L.; McLinden, M. O. NIST Standard Reference Database 23: Reference Fluid Thermodynamic and Transport Properties-REFPROP, Version 8.0. 2007.

(20) Páramo, R.; Zouine, M.; Sobrón, F.; Casanova, C. Oxygenated Gasoline Additives: Saturated Heat Capacities between (277 and 355) K. J. Chem. Eng. Data 2004, 49, 58-61.

(21) Counsell, F..; A. Lee, D.; Martin, F. J. Thermodynamic Properties of Organic Oxygen Compounds. Part XXVI. Diethyl Ether. J. Chem. Soc. Inorg. Phys. Theor. 1971, 0, 313-316.

(22) Ali Raza, M.; Valtz, A.; Coquelet, C.; Hallett, P. D.; Afzal, W. Volumetric Properties of Furan and 2-5-Dimethylfuran in Different Industrial Solvents at Temperatures from 278 to $343 \mathrm{~K}$. J. Chem. Eng. Data 2021, 66, 2666-2680.

(23) R.L. Rowley, W.V. Wilding, J.L. Oscarson, Y. Yang, N.A. Zundel, T.E. Daubert, R.P. Danner DIPPR Data Compilation of Pure Chemical Properties Design Institute for Physical Properties. 2006 Provo, UT: Brigham Young University.

(24) Chorazewski, M.; Postnikov, E. B.; Oster, K.; Polishuk, I. Thermodynamic properties of 1, 2dichloroethane and 1, 2-dibromoethane under elevated pressures: experimental results and 
predictions of a novel DIPPR-based version of FT-EoS, PC-SAFT, and CP-PC-SAFT Ind. Eng. Chem. Res., 2015, 54, 9645-9656

(25) Comelli, F. Excess enthalpies of 1, 3-dioxolane with 1, 2-dichloroethane and 1-bromo-2chloroethane Chim. Ind. (Milan, Italy), 1990, 72, 135-8

(26) Tafat-Igoudjilene, O.; Daoudi, H.; Hassein Bey-Larouci, A.; Aitkaci, A. Volumetric properties of binary liquid mixtures of ketones with chloroalkanes at different temperatures and atmospheric pressure Thermochim. Acta, 2013, 561, 63-71

(27) Klofutar, C.; Paljk, S.; Golc-Teger, S. Partial molar volumes and partial molar expansibilities of cholesterol in some aprotic solvents Thermochim. Acta, 1992, 196, 401-413

(28) Manfredini, M.; Marchetti, A.; Sighinolfi, S.; Tassi, L.; Ulrici, A. ; Vignali, M. Densities and excess molar volumes of binary mixtures containing 1, 2-dichloroethane+ 2-methoxyethanol or 1, 2-dimethoxyethane at different temperatures J. Mol. Liq., 2002, 100, 163-181

(29) Garriga, R.; Andres, Ana Cristina; Perez, P.; Gracia, M. Vapor pressures at several temperatures and excess functions at $298.15 \mathrm{~K}$ of butanone with di-n-propyl ether or diisopropyl ether $\mathrm{J}$. Chem. Eng. Data, 1999, 44, 296-302

(30) Gonzalez-Olmos, R.; Iglesias, M.; Santos, B. M. R. P.; Matted, S. Thermodynamics of oxygenate fuel additives as a function of temperature Phys. Chem. Liq., 2008, 46, 223-237

(31) Meng, X.; Wu, J.; Liu, Z. Viscosity and density measurements of diisopropyl ether and dibutyl ether at different temperatures and pressures J. Chem. Eng. Data, 2009, 54, 2353-2358

(32) Montano, D. F.; Martin, S.; Cea, P.; Lopez, M. C.; Artigas, H. Volumetric, acoustic, and refractive properties of isomeric chlorobutanes with diisopropyl ether J. Chem. Eng. Data, 2010, 55(12), 5953-5959

(33) Pandiyan, V.; Oswal, S. L.; Vasantharani, P. Thermodynamic and acoustic properties of binary mixtures of ethers. III. Diisopropyl ether or oxolane with o-or m-toluidines at 303.15, 313.15 and 323.15 K Thermochim. Acta, 2011, 516, 64-73

(34) Evans, T.W.; Edlund ; K.R. Evans, T. W.; Edlund, K. R. (1936). Tertiary Alkyl Ethers Preparation and Properties Ind.Eng.Chem., 1936, 28, 1186-1188

(35) Landaverde-Cortes, D.C.; Estrada-Baltazar, A.; Iglesias-Silva, G.A.; Hall, K.R. Densities and Viscosities of MTBE+ Heptane or Octane at $\mathrm{p}=0.1 \mathrm{MPa}$ from (273.15 to 363.15) $\mathrm{K}$ J.Chem.Eng.Data, 2007, 52, 1226-1232

(36) Meng, X.; Zheng, P.; Wu, J.; Liu, Z. Density and viscosity measurements of diethyl ether from 243 to $373 \mathrm{~K}$ and up to $20 \mathrm{MPa}$ Fluid Phase Equilib., 2008, 271, 1-5

(37) Oudemans, A.C. Sur la densité, le coefficient de dilatation et l'indice de réfraction de l'éther éthylique Recl. Trav. Chim. Pays-Bas, 1885, 4, 269

(38) Taylor, R. S.; Smith; L. B. Taylor; R. S. ;Smith, L. B. The vapor pressures, densities and some derived quantities for ether at low temperatures J. Am. Chem. Soc. 1922, 44, 2450-2463

(39) Timmermans, J. Measurements of the Density of Liquids at Temperatures Below 0 Sci. Proc. R. Dublin Soc. 1912, 13, 310

(40) Young, S. The vapour-pressures, specific volumes, heats of vaporization, and critical constants of thirty pure substances Sci. Proc. R. Dublin Soc. 1910, 12, 374-443. 
TOC Graphic

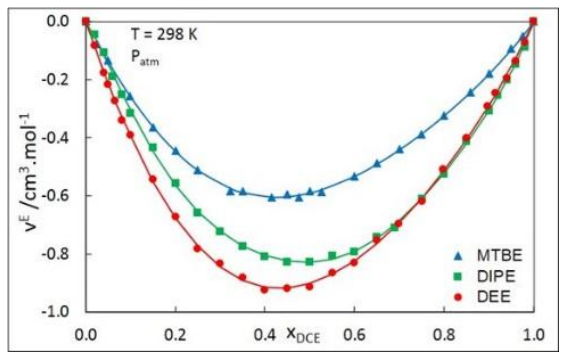

